\title{
Vibration analysis of thin plates resting on Pasternak foundations by element free Galerkin method
}

\author{
Ehsan Bahmyari ${ }^{\mathrm{a}, *}$, Mohammad Mahdi Banatehrani ${ }^{\mathrm{b}}$, Mohammad Ahmadic ${ }^{\mathrm{c}}$ and Marzieh Bahmyari ${ }^{\mathrm{d}}$ \\ ${ }^{a}$ Department of Marine Technology, Amirkabir University of Technology, Tehran, Iran \\ ${ }^{\mathrm{b}}$ Institute for Aerospace Studies, University of Toronto, Toronto, ON, Canada \\ ${ }^{\mathrm{c}}$ Department of Mechanical Engineering, Shahid Chamran University of Ahwaz, Khuzestan, Iran \\ ${ }^{\mathrm{d}}$ Department of Mathematics, Persian Gulf University, Bushehr, Iran
}

Received 23 April 2012

Accepted 3 August 2012

\begin{abstract}
The element free Galerkin method is used to analyze free vibration of thin plates resting on Pasternak elastic foundations with all possible types of classical boundary conditions. Convergence of solution is studied by increasing number of nodes for different boundary conditions and foundation parameters. Upon comparison with available results in literature, it was found that the method converges very fast and has very good accuracy even with small number of nodes. Applicability of the method was shown by solving numerical examples with all possible combinations of boundary conditions and different values of foundation parameters.
\end{abstract}

Keywords: Element free Galerkin method, Pasternak foundation, vibration of plate

\section{Introduction}

Plate is an essential part of structural members in many civil, aeronautical, mechanical and marine structures. Vibration behavior of plates on elastic foundations is of interest for the design of many engineering problems such as pavement of roads, footing of buildings and bases of machines. Winkler type elastic foundation is the simplest model to describe the mechanical behavior of elastic supports. In this model interaction between lateral springs are ignored. Two parameters elastic foundation models have been developed to consider this interaction. They are characterized by two independent elastic constants which are derived by extension of the Winkler's model.

Vibration analysis of plates resting on two parameter elastic foundations was subject of several researches by various approaches. Xiang et al. [1] have studied analytically vibration of rectangular Mindlin plates with simply supported boundary conditions on Pasternak foundation. Omurtag et al. [2] used Finite element method to study the free vibration of thin plates resting on Pasternak foundation. Lam et al. [3] used the Green's functions to derive canonical exact solutions of elastic bending, buckling and vibration for Levy-plates resting on two parameters elastic foundations. Matsunaga [4] developed a two-dimensional higher-order theory to study vibration and stability of thick elastic plates resting on elastic foundation. Shen et al. [5] studied the free and forced vibration of Reissner-Mindlin

\footnotetext{
* Corresponding author: Ehsan Bahmyari, Department of Marine Technology, Amirkabir university of Technology Hafez Ave, No. 424, Tehran, Iran. Tel.: +98 216454 3120; E-mail: Ehsan_Bahmyari@aut.ac.ir.
} 
plates with four free edges resting on a Pasternak elastic foundation by employing the Rayleigh-Ritz method. Zhou et al. [6] used Rayleigh-Ritz method to study the three dimensional vibration of rectangular thick plates on elastic foundations. Ferreira et al. [7] employed the radial basis function collocation method to analysis static deformation and free vibration of plates on Pasternak foundations.

Meshless method is an approximate solution to many boundary value problems which approximations are entirely based on nodal parameters. Varity of meshless methods have been introduced, namely the smoothed particle hydrodynamics (SPH) [8], the meshless local Petrov Galerkin method (MLPG) [9], the reproducing kernel particle method (RKPM) [10], the element free Galerkin method (EFG) [11] and the radial basis function approach [1215]. Element free Galerkin method (EFG) is considered as one of meshless methods which is used for solution of bending, buckling and vibration of plates and shells. Yan et al. [16] have used this method for vibration analysis of isotropic rectangular plate with interior elastic point support and elastically restrained edges. Chen et al. [17] and Dai et al. [18] have used EFG method for vibration analysis of laminated composite plates.

The previous publications have concentrated their studies on limited types of boundary conditions. For example, only one type of boundary condition has been used in References $[1,4,5]$ and also two types of boundary condition in Reference [2], three types of boundary condition in References [6,7] and six types of boundary condition in Reference [3] have been analyzed.

It is the main objective of this paper to use EFG method for vibration analysis of thin plates with all possible types of boundary conditions resting on two parameters elastic foundation. The plate is discretized by a set of regularly distributed nodes. On the basis of classical plate theory (CTP) basic equation of vibration is derived. Moving least square (MLS) approximation is employed to produce displacement shape functions and Penalty method is used for imposing essential boundary conditions. Convergence, accuracy and applicability of the method were demonstrated by vibration solutions of thin plates with general boundary conditions. These important results can be served as benchmark results for researchers to verify their numerical methods and also for engineers to use such plates in their structures in the future.

\section{Moving least square approximation (MLS)}

Moving least square approximation was developed by Lancaster and Salkauskas [19] for surface construction This method has been used widely for generation of shape functions in the meshfree methods. According to the Moving Least Square method, the unknown function $\mathrm{u}(\mathbf{x})$ is approximated by $u^{h}(x)$ as follows:

$$
u^{h}(\mathbf{x})=\sum_{j=1}^{m} p_{j}(\mathbf{x}) a_{j}(\mathbf{x})=\mathbf{p}^{\mathrm{T}}(\mathbf{x}) \mathbf{a}(\mathbf{x})
$$

where $\mathbf{p}(\mathbf{x})$ is a vector of monomial basis functions, and $\mathbf{a}(\mathbf{x})$ is a vector of unknown coefficients which depend on location $x$. Also, $m$ is the numbers of terms in the basis. Commonly used bases are the linear and quadratic basis functions. Quadratic basis in two-dimensional domain has following form:

$$
\mathbf{P}^{\mathrm{T}}=\left[1, x, y, x^{2}, x y, y^{2}\right]
$$

By minimizing weighted square of residual function, the unknown coefficients $a_{j}(x)$ in Eq. (1) can be obtained as follows:

$$
J=\sum_{I=1}^{n} w\left(\mathbf{x}-\mathbf{x}_{I}\right)\left[\mathbf{p}^{\mathrm{T}}\left(\mathbf{x}_{I}\right) \mathbf{a}(\mathbf{x})-u_{I}\right]^{2}
$$

where $u_{I}$ is nodal parameter of field variable at node $I$ and $n$ is the number of nodes in the neighborhood of $\boldsymbol{x}$ which called domain of influence. The unknown coefficients vector $\mathbf{a}(\mathbf{x})$ can be determined by minimizing the functional of the weighted residual as follows:

$$
\frac{\partial J}{\partial \mathbf{a}}=0
$$


Table 1

First and sixth frequency parameters, $\bar{\omega}=\omega a^{2} \sqrt{\frac{\rho h}{D}}$, of a square thin plate resting on Winkler's type elastic foundation

\begin{tabular}{|c|c|c|c|c|c|c|c|c|}
\hline \multirow[t]{2}{*}{$K_{1}$} & \multirow[t]{2}{*}{ Mode number } & \multirow[t]{2}{*}{ Method } & \multicolumn{6}{|c|}{ Natural frequency parameter } \\
\hline & & & SSSS & SCSC & SFSF & SSSC & SCSF & SSSF \\
\hline \multirow[t]{20}{*}{100} & \multirow[t]{10}{*}{ First mode } & Pres. $(5 \times 5)$ & 22.1997 & 39.7748 & 13.9200 & 27.7797 & 16.6801 & 15.4248 \\
\hline & & Pres. $(7 \times 7)$ & 22.1388 & 31.0169 & 13.8917 & 25.9713 & 16.1896 & 15.3891 \\
\hline & & Pres. $(9 \times 9)$ & 22.1380 & 30.8754 & 13.8955 & 25.7790 & 16.1742 & 15.3900 \\
\hline & & Pres. $(11 \times 11)$ & 22.1385 & 30.8351 & 13.8943 & 25.7450 & 16.1697 & 15.3889 \\
\hline & & Pres. $(13 \times 13)$ & 22.1363 & 30.7795 & 13.8919 & 25.7246 & 16.1655 & 15.3867 \\
\hline & & Pres. $(15 \times 15)$ & 22.1342 & 30.7364 & 13.8901 & 25.7121 & 16.1628 & 15.3850 \\
\hline & & Pres. $(17 \times 17)$ & 22.1326 & 30.7141 & 13.8888 & 25.7041 & 16.1611 & 15.3838 \\
\hline & & Pres. $(19 \times 19)$ & 22.1315 & 30.6992 & 13.8879 & 25.6983 & 16.1598 & 15.3829 \\
\hline & & Pres. $(23 \times 23)$ & 22.1299 & 30.6786 & 13.8866 & 25.6906 & 16.1581 & 15.3817 \\
\hline & & Ref [3] & 22.13 & 30.63 & 13.88 & 25.67 & 16.15 & 15.38 \\
\hline & \multirow[t]{10}{*}{ Sixth mode } & Pres. $(5 \times 5)$ & 221.2307 & $2.6824 \times 10^{3}$ & 81.4949 & 559.4443 & 159.4388 & 158.5789 \\
\hline & & Pres. $(7 \times 7)$ & 103.3783 & 145.4672 & 71.6906 & 121.7760 & 95.7161 & 95.2384 \\
\hline & & Pres. $(9 \times 9)$ & 101.3152 & 132.1131 & 71.6137 & 116.2383 & 92.9194 & 92.6162 \\
\hline & & Pres. $(11 \times 11)$ & 100.0937 & 130.9692 & 71.5525 & 114.8928 & 92.0405 & 91.7153 \\
\hline & & Pres. $(13 \times 13)$ & 99.7609 & 130.9690 & 71.5187 & 114.6726 & 91.7934 & 91.4683 \\
\hline & & Pres. $(15 \times 15)$ & 99.6474 & 130.8205 & 71.5015 & 114.5373 & 91.6701 & 91.3523 \\
\hline & & Pres. $(17 \times 17)$ & 99.5727 & 130.6771 & 71.4892 & 114.4154 & 91.5865 & 91.2701 \\
\hline & & Pres. $(19 \times 19)$ & 99.5162 & 130.5475 & 71.4791 & 114.3138 & 91.5233 & 91.2069 \\
\hline & & Pres. $(23 \times 23)$ & 99.4325 & 130.3091 & 71.4642 & 114.1546 & 91.4292 & 91.1130 \\
\hline & & Ref [3] & 99.20 & 129.48 & 71.44 & 113.67 & 91.16 & 90.85 \\
\hline \multirow[t]{20}{*}{1000} & \multirow[t]{10}{*}{ First mode } & Pres. $(5 \times 5)$ & 37.3263 & 51.5988 & 33.0712 & 41.2286 & 34.3728 & 33.7327 \\
\hline & & Pres. $(7 \times 7)$ & 37.2827 & 43.1561 & 33.0591 & 39.6853 & 34.0886 & 33.7155 \\
\hline & & Pres. $(9 \times 9)$ & 37.2820 & 43.0487 & 33.0607 & 39.5528 & 34.0809 & 33.7158 \\
\hline & & Pres. $(11 \times 11)$ & 37.2823 & 43.0193 & 33.0602 & 39.5304 & 34.0787 & 33.7153 \\
\hline & & Pres. $(13 \times 13)$ & 37.2809 & 42.9792 & 33.0592 & 39.5171 & 34.0767 & 33.7143 \\
\hline & & Pres. $(15 \times 15)$ & 37.2797 & 42.9483 & 33.0584 & 39.5089 & 34.0754 & 33.7135 \\
\hline & & Pres. $(17 \times 17)$ & 37.2787 & 42.9324 & 33.0579 & 39.5037 & 34.0746 & 33.7130 \\
\hline & & Pres. $(19 \times 19)$ & 37.2781 & 42.9217 & 33.0575 & 39.4999 & 34.0740 & 33.7126 \\
\hline & & Pres. $(23 \times 23)$ & 37.2771 & 42.9070 & 33.0570 & 39.4949 & 34.0732 & 33.7120 \\
\hline & & Ref [3] & 37.28 & 42.87 & 31.62 & 39.49 & 34.07 & 33.71 \\
\hline & \multirow[t]{10}{*}{ Sixth mode } & Pres. $(5 \times 5)$ & 238.9642 & $3.0594 \times 10^{3}$ & 86.8640 & 630.8066 & 162.3892 & 161.5506 \\
\hline & & Pres. $(7 \times 7)$ & 107.6658 & 148.6465 & 77.7132 & 125.4638 & 100.3083 & 99.8551 \\
\hline & & Pres. $(9 \times 9)$ & 105.6708 & 135.4854 & 77.6402 & 120.0558 & 97.6396 & 97.3515 \\
\hline & & Pres. $(11 \times 11)$ & 104.4904 & 134.3589 & 77.5831 & 118.7426 & 96.8028 & 96.4937 \\
\hline & & Pres. $(13 \times 13)$ & 104.1706 & 134.3583 & 77.5518 & 118.5287 & 96.5678 & 96.2588 \\
\hline & & Pres. $(15 \times 15)$ & 104.0618 & 134.2132 & 77.5360 & 118.3977 & 96.4506 & 96.1486 \\
\hline & & Pres. $(17 \times 17)$ & 103.9903 & 134.0734 & 77.5246 & 18.2798 & 96.3711 & 96.0705 \\
\hline & & Pres. $(19 \times 19)$ & 103.9362 & 133.9471 & 77.5153 & 118.1815 & 96.3110 & 96.0105 \\
\hline & & Pres. $(23 \times 23)$ & 103.8561 & 133.7148 & 77.5016 & 118.0275 & 96.2216 & 95.9213 \\
\hline & & Ref [3] & 103.64 & 132.91 & 77.49 & 117.56 & 95.97 & 95.67 \\
\hline
\end{tabular}

which yields following system of linear equations:

$$
\mathbf{A}(\mathbf{x}) \mathbf{a}(\mathbf{x})=\mathbf{B}(\mathbf{x}) \mathbf{U}_{s}
$$

where,

$$
\begin{aligned}
& \mathbf{A}(\mathbf{x})=\sum_{I=1}^{n} w\left(\mathbf{x}-\mathbf{x}_{I}\right) \mathbf{p}\left(\mathbf{x}_{I}\right) \mathbf{p}^{\mathrm{T}}\left(\mathbf{x}_{I}\right) \\
& \mathbf{B}(\mathbf{x})=\left[\begin{array}{ll}
w\left(\mathbf{x}-\mathbf{x}_{I}\right) \mathbf{p}\left(\mathbf{x}_{I}\right), \ldots, w\left(\mathbf{x}-\mathbf{x}_{n}\right) \mathbf{p}\left(\mathbf{x}_{n}\right)
\end{array}\right] \\
& \mathbf{U}_{s}=\left\{\begin{array}{llll}
u_{1} & u_{2} & \ldots & u_{n}
\end{array}\right\}^{\mathrm{T}}
\end{aligned}
$$

By substituting Eq. (5) into Eq. (1), MLS approximant can be written as:

$$
u^{h}(\mathbf{x})=\sum_{I=1}^{n} \phi_{I}(\mathbf{x}) u_{I}=\boldsymbol{\Phi}(\mathbf{x}) \mathbf{U}_{s}
$$




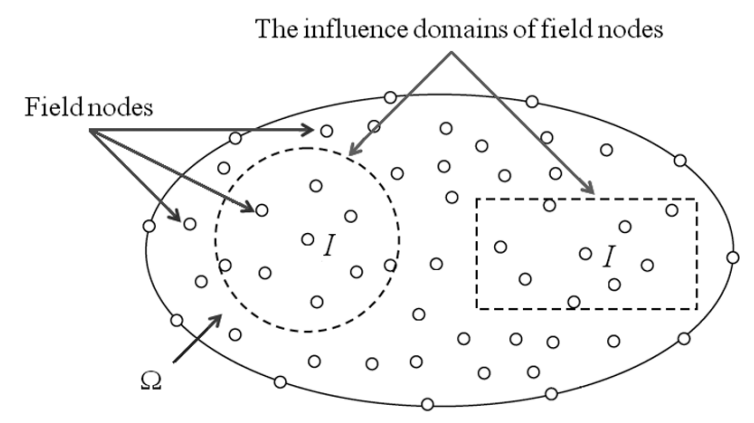

Fig. 1. Domain of influence.

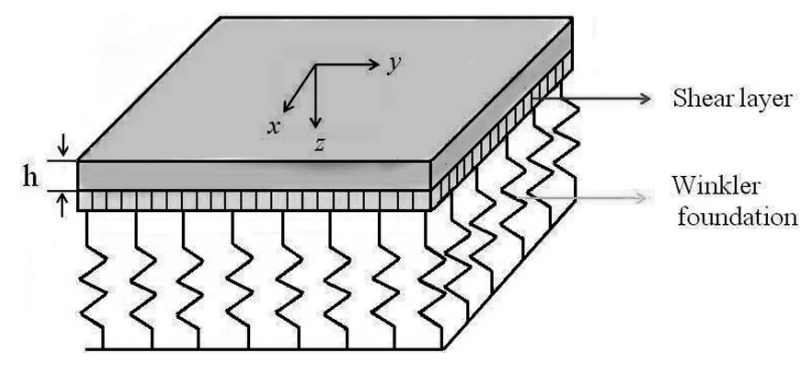

Fig. 2. Thin plate resting on two parameter elastic foundation and coordinate system.

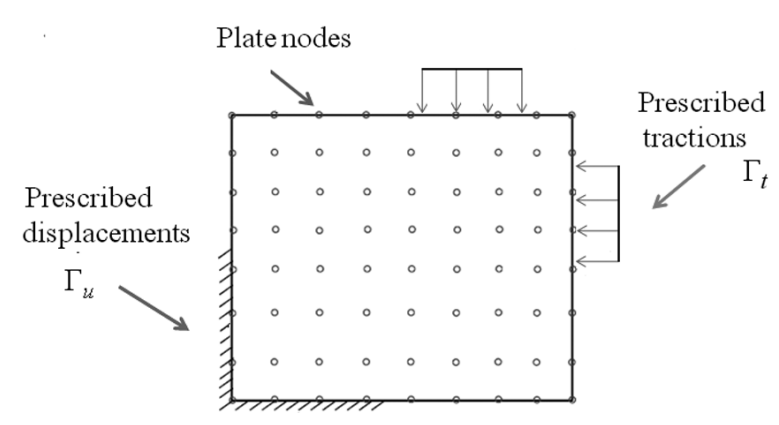

Fig. 3. Prescribed boundary conditions.

where $\boldsymbol{\Phi}(\mathbf{x})$ is matrix of shape functions and is defined as follows:

$$
\boldsymbol{\Phi}(\mathbf{x})=\left[\phi_{1}(\mathbf{x}) \phi_{2}(\mathbf{x}) \ldots \phi_{n}(\mathbf{x})\right]=\mathbf{p}^{\mathrm{T}}(\mathbf{x}) \mathbf{A}^{-1}(\mathbf{x}) \mathbf{B}(\mathbf{x})
$$

In order to calculate partial derivatives of $\boldsymbol{\Phi}(\mathbf{x})$, Eq. (10) is rewritten as follows [20]:

$$
\mathbf{\Phi}(\mathbf{x})=\gamma^{\mathrm{T}}(\mathbf{x}) \mathbf{B}(\mathbf{x})
$$

where

$$
\gamma(\mathbf{x})=\mathbf{A}^{-1}(\mathbf{x}) \mathbf{p}(\mathbf{x})
$$

or,

$$
\mathbf{A}(\mathbf{x}) \gamma(\mathbf{x})=\mathbf{p}(\mathbf{x})
$$

The partial derivatives of $\gamma(\mathbf{x})$ can be expressed as follows:

$$
\begin{aligned}
\mathbf{A} \gamma_{, x} & =\mathbf{p}_{, x}-\mathbf{A}_{, x} \gamma \\
\mathbf{A} \gamma_{, y} & =\mathbf{p}_{, y}-\mathbf{A}_{, y} \gamma \\
\mathbf{A} \gamma_{, x x} & =\mathbf{p}_{x x}-\left(\mathbf{A}_{, x x} \gamma+2 \mathbf{A}_{, x} \boldsymbol{\gamma}_{, x}\right) \\
\mathbf{A} \gamma_{, x y} & =\mathbf{p}_{, x y}-\left(\mathbf{A}_{, x y} \gamma+\mathbf{A}_{, x} \boldsymbol{\gamma}_{, y}+\mathbf{A}_{, y} \boldsymbol{\gamma}_{, x}\right) \\
\mathbf{A} \gamma_{, y y} & =\mathbf{p}_{, y y}-\left(\mathbf{A}_{, y y} \gamma+2 \mathbf{A}_{, y} \boldsymbol{\gamma}_{, y}\right)
\end{aligned}
$$


Table 2

Dimensionless parameter of natural frequencies, $\bar{\omega}=\frac{\omega a^{2}}{\pi^{2}} \sqrt{\frac{\rho h}{D}}$, of a square SSSS plate resting on Winkler's type elastic foundation

\begin{tabular}{|c|c|c|c|c|c|c|c|}
\hline \multirow[t]{2}{*}{$\mathrm{K}_{1}$} & \multirow[t]{2}{*}{ Method } & \multicolumn{6}{|c|}{ Mode number } \\
\hline & & $1 \mathrm{st}$ & 2nd & $3 \mathrm{rd}$ & 4th & 5 th & 6th \\
\hline \multirow[t]{12}{*}{100} & Pres. $(5 \times 5)$ & 2.2493 & 6.8840 & 6.8840 & 15.6987 & 16.2849 & 22.4154 \\
\hline & Pres. $(7 \times 7)$ & 2.2431 & 5.2509 & 5.2509 & 8.4292 & 10.4681 & 10.4744 \\
\hline & Pres. $(9 \times 9)$ & 2.2430 & 5.1231 & 5.1231 & 8.0986 & 10.2630 & 10.2654 \\
\hline & Pres. $(11 \times 11)$ & 2.2431 & 5.1153 & 5.1153 & 8.0797 & 10.1380 & 10.1416 \\
\hline & Pres. $(13 \times 13)$ & 2.2429 & 5.1125 & 5.1125 & 8.0752 & 10.1074 & 10.1079 \\
\hline & Pres. $(15 \times 15)$ & 2.2427 & 5.1107 & 5.1107 & 8.0730 & 10.0961 & 10.0964 \\
\hline & Pres. $(17 \times 17)$ & 2.2425 & 5.1092 & 5.1092 & 8.0714 & 10.0886 & 10.0888 \\
\hline & Pres. $(19 \times 19)$ & 2.2424 & 5.1079 & 5.1079 & 8.0700 & 10.0830 & 10.0831 \\
\hline & Pres. $(23 \times 23)$ & 2.2422 & 5.1059 & 5.1059 & 8.0678 & 10.0746 & 10.0746 \\
\hline & Ref. [1] & 2.2413 & 5.0971 & 5.0971 & 8.0523 & & \\
\hline & Ref. [6] & 2.2413 & 5.0973 & 5.0973 & 8.0527 & & \\
\hline & Ref. [7] & 2.2414 & 5.0967 & 5.0967 & 8.0542 & & \\
\hline \multirow[t]{12}{*}{500} & Pres. $(5 \times 5)$ & 3.0278 & 7.2613 & 7.2613 & 15.8295 & 17.1184 & 23.2311 \\
\hline & Pres. $(7 \times 7)$ & 3.0228 & 5.6306 & 5.6306 & 8.6774 & 10.6635 & 10.6697 \\
\hline & Pres. $(9 \times 9)$ & 3.0227 & 5.5092 & 5.5092 & 8.3483 & 10.4615 & 10.4638 \\
\hline & Pres. $(11 \times 11)$ & 3.0228 & 5.5020 & 5.5020 & 8.3298 & 10.3384 & 10.3420 \\
\hline & Pres. $(13 \times 13)$ & 3.0226 & 5.4993 & 5.4993 & 8.3254 & 10.3084 & 10.3089 \\
\hline & Pres. $(15 \times 15)$ & 3.0225 & 5.4976 & 5.4976 & 8.3233 & 10.2973 & 10.2976 \\
\hline & Pres. $(17 \times 17)$ & 3.0223 & 5.4962 & 5.4962 & 8.3217 & 10.2900 & 10.2902 \\
\hline & Pres. $(19 \times 19)$ & 3.0223 & 5.4950 & 5.4950 & 8.3203 & 10.2844 & 10.2845 \\
\hline & Pres. $(23 \times 23)$ & 3.0221 & 5.4932 & 5.4932 & 8.3182 & 10.2762 & 10.2762 \\
\hline & Ref. [1] & 3.0215 & 5.4850 & 5.4850 & 8.3032 & & \\
\hline & Ref. [6] & 3.0214 & 5.4850 & 5.4850 & 8.3035 & & \\
\hline & Ref. [7] & 3.0216 & 5.4846 & 5.4846 & 8.3051 & & \\
\hline
\end{tabular}

The partial derivatives of $\mathbf{\Phi}(\mathbf{x})$ would be as follows:

$$
\begin{aligned}
\boldsymbol{\Phi}_{I, x} & =\gamma_{, x}^{\mathrm{T}} \mathbf{B}_{I}+\gamma^{\mathrm{T}} \mathbf{B}_{I, x} \\
\boldsymbol{\Phi}_{I, y} & =\gamma_{, y}^{\mathrm{T}} \mathbf{B}_{I}+\gamma^{\mathrm{T}} \mathbf{B}_{I, y} \\
\boldsymbol{\Phi}_{I, x x} & =\gamma_{, x x}^{\mathrm{T}} \mathbf{B}_{I}+2 \boldsymbol{\gamma}_{, x}^{\mathrm{T}} \mathbf{B}_{I, x}+\gamma^{\mathrm{T}} \mathbf{B}_{I, x x} \\
\boldsymbol{\Phi}_{I, x y} & =\gamma_{, x y}^{\mathrm{T}} \mathbf{B}_{I}+\boldsymbol{\gamma}_{, x}^{\mathrm{T}} \mathbf{B}_{I, y}+\gamma_{, y}^{\mathrm{T}} \mathbf{B}_{I, x}+\gamma^{\mathrm{T}} \mathbf{B}_{I, x y} \\
\boldsymbol{\Phi}_{I, y y} & =\gamma_{, y y}^{\mathrm{T}} \mathbf{B}_{I}+2 \boldsymbol{\gamma}_{, y}^{\mathrm{T}} \mathbf{B}_{I, y}+\gamma^{\mathrm{T}} \mathbf{B}_{I, y y}
\end{aligned}
$$

Weight function plays an important role in the formulation of MLS method. This function should be non-zero in the domain of influence and zero outside of the domain. The precise character of this function seems to be unimportant although it is almost mandatory that it be positive and increase monotonically as $\left\|x-x_{I}\right\|$ decreases, furthermore it is desirable that weight function be smooth [21]. Several weight functions have been proposed by researchers. In this work quartic spline weight function is chosen,

$$
w\left(\mathbf{x}-\mathbf{x}_{I}\right) \equiv w(r)= \begin{cases}1-6 r^{2}+8 r^{3}-3 r^{4}, & r \leqslant 1 \\ 0, & r>1\end{cases}
$$

where,

$$
r=\frac{\left\|\mathbf{x}-\mathbf{x}_{I}\right\|}{d_{I}}
$$

where $d_{I}$ determines the size of the influence domain at node $I$. The most commonly used domains are circles and rectangles (Fig. 1). For circular domain, $d_{I}$ is the radius of circle and for rectangular domain, $d_{I}$ is equal to length of rectangle in $x$, and $y$ direction which are denoted by $d_{I x}$ and $d_{I y}$, respectively. For the latter case, weight function can be written as follows:

$$
w\left(\mathbf{x}-\mathbf{x}_{I}\right)=w\left(r_{x}\right) \cdot w\left(r_{y}\right)
$$




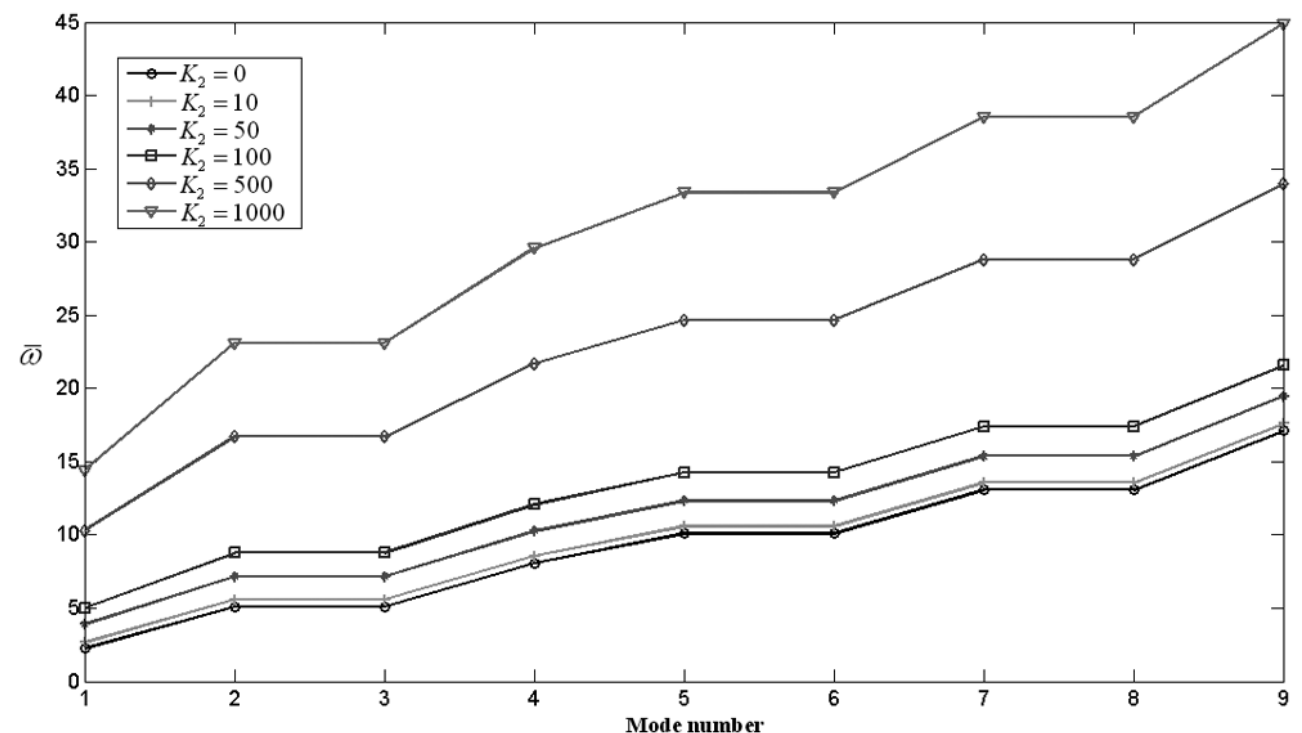

Fig. 4. Comparison of natural frequencies for Winkler and Pasternak's type of elastic foundation $\left(K_{1}=100, h / a=0.01\right.$ and number of nodes $19 \times 19)$.

where $r_{x}$ and $r_{y}$ are given by

$$
\begin{aligned}
& r_{x}=\frac{\left\|x-x_{I}\right\|}{d_{I x}} \\
& r_{y}=\frac{\left\|y-y_{I}\right\|}{d_{I y}}
\end{aligned}
$$

where

$$
\begin{aligned}
& d_{I x}=d_{\max } \cdot c_{I x} \\
& d_{I y}=d_{\max } \cdot c_{I y}
\end{aligned}
$$

where $d_{\max }$ is scaling parameter, $c_{I x}$ and $c_{I y}$ are distance around node $I$ which are determined in such a way that enough nodes be in the domain in order that matrix $\mathbf{A}$ in Eq. (13) to be invertible at every point in the domain [21].

\section{Governing equations}

\subsection{Strain and kinetic energies of thin plate}

A thin plate of thickness $h$ resting on a two-parametric elastic foundation with Cartesian coordinate system origin at center is shown in Fig. 2. Displacements functions in $x, y$ and $z$ directions are defined by $u, v$ and $w$, respectively. Based on CPT, displacement field can be expressed as follows [17]:

$$
\mathbf{u}=\left\{\begin{array}{l}
u \\
v \\
w
\end{array}\right\}=\left\{-z \frac{\partial}{\partial x}-z \frac{\partial}{\partial y} 1\right\}^{\mathrm{T}} w=\mathbf{L}_{u} w
$$


Table 3

First and sixth frequency parameters, $\bar{\omega}=\omega a^{2} \sqrt{\frac{\rho h}{D}}$, of a square thin plate resting on Pasternak's type elastic foundation

\begin{tabular}{|c|c|c|c|c|c|c|c|c|c|}
\hline \multirow[t]{2}{*}{$\mathrm{K}_{1}$} & \multirow[t]{2}{*}{$\mathrm{K}_{2}$} & \multirow[t]{2}{*}{ Mode number } & \multirow[t]{2}{*}{ Method } & \multicolumn{6}{|c|}{ Natural frequency parameter } \\
\hline & & & & SSSS & SCSC & SFSF & SSSC & SCSF & SSSF \\
\hline \multirow[t]{20}{*}{0} & \multirow[t]{20}{*}{0} & \multirow[t]{10}{*}{ First mode } & Pres. $(5 \times 5)$ & 19.8187 & 38.2349 & 9.6837 & 25.8562 & 13.3355 & 11.7442 \\
\hline & & & Pres. $(7 \times 7)$ & 19.7520 & 29.3599 & 9.6430 & 23.9680 & 12.7323 & 11.6976 \\
\hline & & & Pres. $(9 \times 9)$ & 19.7511 & 29.2113 & 9.6485 & 23.7607 & 12.7129 & 11.6989 \\
\hline & & & Pres. $(11 \times 11)$ & 19.7517 & 29.1688 & 9.6468 & 23.7239 & 12.7072 & 11.6973 \\
\hline & & & Pres. $(13 \times 13)$ & 19.7492 & 29.1100 & 9.6433 & 23.7018 & 12.7018 & 11.6945 \\
\hline & & & Pres. $(15 \times 15)$ & 19.7468 & 29.0644 & 9.6407 & 23.6882 & 12.6984 & 11.6923 \\
\hline & & & Pres. $(17 \times 17)$ & 19.7451 & 29.0409 & 9.6388 & 23.6795 & 12.6962 & 11.6907 \\
\hline & & & Pres. $(19 \times 19)$ & 19.7438 & 29.0251 & 9.6375 & 23.6732 & 12.6946 & 11.6895 \\
\hline & & & Pres. $(23 \times 23)$ & 19.7421 & 29.0033 & 9.6356 & 23.6649 & 12.6923 & 11.6879 \\
\hline & & & Ref [3] & 19.74 & 28.95 & 9.63 & 23.65 & 12.69 & 11.68 \\
\hline & & \multirow[t]{10}{*}{ Sixth mode } & Pres. $(5 \times 5)$ & 219.1715 & $2.6372 \times 10^{3}$ & 80.8759 & 550.9448 & 159.1064 & 158.2405 \\
\hline & & & Pres. $(7 \times 7)$ & 102.8908 & 145.1095 & 70.9898 & 121.3593 & 95.1922 & 94.7116 \\
\hline & & & Pres. $(9 \times 9)$ & 100.8196 & 131.7330 & 70.9124 & 115.8063 & 92.3800 & 92.0751 \\
\hline & & & Pres. $(11 \times 11)$ & 99.5932 & 130.5871 & 70.8508 & 114.4571 & 91.4960 & 91.1689 \\
\hline & & & Pres. $(13 \times 13)$ & 99.2588 & 130.5870 & 70.8166 & 114.2361 & 91.2475 & 90.9204 \\
\hline & & & Pres. $(15 \times 15)$ & 99.1447 & 130.4380 & 70.7993 & 114.1003 & 91.1234 & 90.8037 \\
\hline & & & Pres. $(17 \times 17)$ & 99.0697 & 130.2942 & 70.7868 & 113.9779 & 91.0393 & 90.7211 \\
\hline & & & Pres. $(19 \times 19)$ & 99.0129 & 130.1643 & 70.7767 & 113.8760 & 90.9757 & 90.6575 \\
\hline & & & Pres. $(23 \times 23)$ & 98.9288 & 129.9252 & 70.7616 & 113.7161 & 90.8811 & 90.5630 \\
\hline & & & Ref [3] & 98.69 & 129.09 & 70.74 & 113.23 & 90.61 & 90.29 \\
\hline \multirow[t]{20}{*}{0} & \multirow[t]{20}{*}{100} & \multirow[t]{10}{*}{ First mode } & Pres. $(5 \times 5)$ & 48.6709 & 65.9214 & 32.9237 & 54.0423 & 38.8593 & 37.1864 \\
\hline & & & Pres. $(7 \times 7)$ & 48.6194 & 55.1007 & 32.9062 & 51.5800 & 38.0427 & 37.1545 \\
\hline & & & Pres. $(9 \times 9)$ & 48.6183 & 54.8322 & 32.9083 & 51.4035 & 37.9990 & 37.1548 \\
\hline & & & Pres. $(11 \times 11)$ & 48.6188 & 54.8028 & 32.9080 & 51.3710 & 37.9905 & 37.1545 \\
\hline & & & Pres. $(13 \times 13)$ & 48.6177 & 54.7798 & 32.9071 & 51.3577 & 37.9869 & 37.1536 \\
\hline & & & Pres. $(15 \times 15)$ & 48.6167 & 54.7552 & 32.9063 & 51.3501 & 37.9850 & 37.1529 \\
\hline & & & Pres. $(17 \times 17)$ & 48.6160 & 54.7431 & 32.9057 & 51.3452 & 37.9836 & 37.1524 \\
\hline & & & Pres. $(19 \times 19)$ & 48.6154 & 54.7349 & 32.9053 & 51.3414 & 37.9825 & 37.1520 \\
\hline & & & Pres. $(23 \times 23)$ & 48.6146 & 54.7225 & 32.9047 & 51.3359 & 37.9809 & 37.1515 \\
\hline & & & $\operatorname{Ref}[3]$ & 48.62 & 54.68 & 32.90 & 51.32 & 37.98 & 37.15 \\
\hline & & \multirow[t]{10}{*}{ Sixth mode } & Pres. $(5 \times 5)$ & 298.1788 & $3.9594 \times 10^{3}$ & 126.8551 & 810.8308 & 210.1817 & 191.3902 \\
\hline & & & Pres. $(7 \times 7)$ & 143.6404 & 178.7094 & 115.5538 & 158.8996 & 135.7528 & 135.2073 \\
\hline & & & Pres. $(9 \times 9)$ & 141.7370 & 167.9889 & 115.4758 & 154.3975 & 133.3230 & 132.9989 \\
\hline & & & Pres. $(11 \times 11)$ & 140.6843 & 166.8371 & 115.3670 & 153.1456 & 132.5973 & 132.2724 \\
\hline & & & Pres. $(13 \times 13)$ & 140.4231 & 166.8450 & 115.3114 & 152.9621 & 132.4086 & 132.0906 \\
\hline & & & Pres. $(15 \times 15)$ & 140.3387 & 166.7491 & 115.2892 & 152.8701 & 132.3183 & 132.0076 \\
\hline & & & Pres. $(17 \times 17)$ & 140.2832 & 166.6527 & 115.2765 & 152.7868 & 132.2575 & 131.9487 \\
\hline & & & Pres. $(19 \times 19)$ & 140.2413 & 166.5637 & 115.2671 & 152.7155 & 132.2117 & 131.9033 \\
\hline & & & Pres. $(23 \times 23)$ & 140.1794 & 166.3948 & 115.2541 & 152.6009 & 132.1440 & 131.8361 \\
\hline & & & Ref [3] & 140.04 & 165.75 & 115.25 & 152.24 & 131.98 & 131.67 \\
\hline \multirow[t]{15}{*}{100} & \multirow[t]{15}{*}{100} & \multirow[t]{10}{*}{ First mode } & Pres. $(5 \times 5)$ & 49.6881 & 66.8246 & 34.4088 & 54.9899 & 40.1342 & 38.5076 \\
\hline & & & Pres. $(7 \times 7)$ & 49.6370 & 56.0014 & 34.3920 & 52.5408 & 39.3351 & 38.4766 \\
\hline & & & Pres. $(9 \times 9)$ & 49.6359 & 55.7365 & 34.3941 & 52.3671 & 39.2927 & 38.4768 \\
\hline & & & Pres. $(11 \times 11)$ & 49.6364 & 55.7075 & 34.3938 & 52.3351 & 39.2845 & 38.4765 \\
\hline & & & Pres. $(13 \times 13)$ & 49.6353 & 55.6849 & 34.3928 & 52.3220 & 39.2810 & 38.4757 \\
\hline & & & Pres. $(15 \times 15)$ & 49.6344 & 55.6607 & 34.3921 & 52.3146 & 39.2791 & 38.4751 \\
\hline & & & Pres. $(17 \times 17)$ & 49.6336 & 55.6488 & 34.3915 & 52.3098 & 39.2778 & 38.4746 \\
\hline & & & Pres. $(19 \times 19)$ & 49.6331 & 55.6407 & 34.3911 & 52.3060 & 39.2767 & 38.4742 \\
\hline & & & Pres. $(23 \times 23)$ & 49.6323 & 55.6285 & 34.3905 & 52.3006 & 39.2752 & 38.4737 \\
\hline & & & $\operatorname{Ref}[3]$ & 49.63 & 55.59 & 34.39 & 52.29 & 39.27 & 38.47 \\
\hline & & Sixth mode & Pres. $(5 \times 5)$ & 299.6952 & $3.9897 \times 10^{3}$ & 127.2539 & 816.6297 & 210.5833 & 191.6662 \\
\hline & & & Pres. $(7 \times 7)$ & 143.9899 & 178.9988 & 115.9859 & 159.2181 & 136.1206 & 135.5767 \\
\hline & & & Pres. $(9 \times 9)$ & 142.0899 & 168.2871 & 115.9078 & 154.7217 & 133.6973 & 133.3742 \\
\hline & & & Pres. $(11 \times 11)$ & 141.0391 & 167.1364 & 115.7994 & 153.4715 & 132.9735 & 132.6496 \\
\hline & & & Pres. $(13 \times 13)$ & 140.7784 & 167.1442 & 115.7439 & 153.2884 & 132.7854 & 132.4683 \\
\hline
\end{tabular}


Table 3, continued

\begin{tabular}{|c|c|c|c|c|c|c|c|c|c|}
\hline \multirow[t]{2}{*}{$\mathrm{K}_{1}$} & \multirow[t]{2}{*}{$\mathrm{K}_{2}$} & \multirow[t]{2}{*}{ Mode number } & \multirow[t]{2}{*}{ Method } & \multicolumn{6}{|c|}{ Natural frequency parameter } \\
\hline & & & & SSSS & SCSC & SFSF & SSSC & SCSF & SSSF \\
\hline & & & Pres. $(15 \times 15)$ & 140.6942 & 167.0484 & 115.7218 & 153.1965 & 132.6953 & 132.3856 \\
\hline & & & Pres. $(17 \times 17)$ & 49.6336 & 166.9522 & 115.7091 & 153.1135 & 132.6348 & 132.3268 \\
\hline & & & Pres. $(19 \times 19)$ & 140.5970 & 166.8633 & 115.6998 & 153.0423 & 132.5891 & 132.2815 \\
\hline & & & Pres. $(23 \times 23)$ & 140.5353 & 166.6948 & 115.6868 & 152.9279 & 132.5215 & 132.2145 \\
\hline & & & $\operatorname{Ref}[3]$ & 140.39 & 166.05 & 115.69 & 152.57 & 132.36 & 132.05 \\
\hline
\end{tabular}

The pseudo-strains of the plate are denoted as

$$
\varepsilon_{p}=\left\{-\frac{\partial^{2}}{\partial x^{2}}-\frac{\partial^{2}}{\partial y^{2}}-2 \frac{\partial^{2}}{\partial x \partial y}\right\}^{\mathrm{T}} w=\mathbf{L}_{p s} w
$$

The pseudo-stresses of the plate are given by

$$
\boldsymbol{\sigma}_{p}=\left\{\begin{array}{l}
M_{x} \\
M_{y} \\
M_{x y}
\end{array}\right\}
$$

The relationship between the pseudo-strains and pseudo-stress is expressed as

$$
\boldsymbol{\sigma}_{p}=\mathbf{D} \varepsilon_{p}
$$

where $\mathbf{D}$ is stiffness matrix and is given as,

$$
\mathbf{D}=\left[\begin{array}{lll}
D_{11} & D_{12} & D_{16} \\
D_{12} & D_{22} & D_{26} \\
D_{16} & D_{26} & D_{66}
\end{array}\right]
$$

For an isotropic plate, D would be as follows:

$$
\mathbf{D}=\frac{\mathrm{Eh}^{3}}{12\left(1-v^{2}\right)}\left[\begin{array}{llc}
1 & v & 0 \\
v & 1 & 0 \\
0 & 0 & (1-v) / 2
\end{array}\right]
$$

where E is Young's module and $v$ is Poisson's ratio. The strain and kinetic energies of the plate can be written as:

$$
\begin{aligned}
& U_{p}=\frac{1}{2} \int_{S} \varepsilon_{P}^{\mathrm{T}} \boldsymbol{\sigma}_{P} \mathrm{~d} S \\
& T_{p}=\frac{1}{2} \int_{V} \rho \dot{\mathbf{u}}^{\mathrm{T}} \dot{\mathbf{u}} \mathrm{d} V
\end{aligned}
$$

\subsection{Strain energy due to elastic foundation}

The surface pressure vector induce by foundation is defined as follows;

$$
\mathbf{q}=\left\{\begin{array}{lll}
0 & 0 & k_{f} w-G_{f} \\
\nabla^{2} w
\end{array}\right\}^{\mathrm{T}}
$$

where $k_{f}$ is the Winkler foundation stiffness while $G_{f}$ is the shear stiffness of the elastic foundation. The strain energy due to the Pasternak foundation is given as follows,

$$
U_{F}=\frac{1}{2} \int_{S} \mathbf{u}^{\mathrm{T}} \mathbf{q} \mathrm{d} S=\frac{1}{2} \int_{S} \boldsymbol{\varepsilon}_{F}^{\mathrm{T}} \boldsymbol{\sigma}_{F} \mathrm{~d} S
$$


Table 4

Dimensionless parameter of natural frequencies, $\bar{\omega}=\frac{\omega a^{2}}{\pi^{2}} \sqrt{\frac{\rho h}{D}}$, of a square SSSS plate resting on Pasternak's type elastic foundation (K $\left.2=10\right)$

\begin{tabular}{|c|c|c|c|c|c|c|c|c|}
\hline \multirow[t]{2}{*}{$\mathrm{h} / \mathrm{a}$} & \multirow[t]{2}{*}{$\mathrm{K}_{1}$} & \multirow[t]{2}{*}{ Method } & \multicolumn{6}{|c|}{ Mode number } \\
\hline & & & $1 \mathrm{st}$ & 2nd & 3rd & 4th & 5 th & 6th \\
\hline \multirow[t]{24}{*}{0.01} & 100 & Pres. $(5 \times 5)$ & 2.6624 & 7.3457 & 7.3457 & 16.0736 & 17.1893 & 23.3326 \\
\hline & & Pres. $(7 \times 7)$ & 2.6567 & 5.7185 & 5.7185 & 8.9137 & 10.9498 & 10.9558 \\
\hline & & Pres. $(9 \times 9)$ & 2.6566 & 5.5958 & 5.5958 & 8.5849 & 10.7480 & 10.7502 \\
\hline & & Pres. $(11 \times 11)$ & 2.6566 & 5.5886 & 5.5886 & 8.5665 & 10.6261 & 10.6296 \\
\hline & & Pres. $(13 \times 13)$ & 2.6564 & 5.5860 & 5.5860 & 8.5622 & 10.5966 & 10.5971 \\
\hline & & Pres. $(15 \times 15)$ & 2.6563 & 5.5843 & 5.5843 & 8.5601 & 10.5858 & 10.5861 \\
\hline & & Pres. $(17 \times 17)$ & 2.6561 & 5.5829 & 5.5829 & 8.5585 & 10.5786 & 10.5788 \\
\hline & & Pres. $(19 \times 19)$ & 2.6560 & 5.5817 & 5.5817 & 8.5572 & 10.5732 & 10.5733 \\
\hline & & Pres. $(23 \times 23)$ & 2.6559 & 5.5799 & 5.5799 & 8.5551 & 10.5651 & 10.5652 \\
\hline & & Ref. [1] & 2.6551 & 5.5718 & 5.5718 & 8.5405 & & \\
\hline & & Ref. [6] & 2.6551 & 5.5717 & 5.5717 & 8.5406 & & \\
\hline & & Ref. [7] & 2.6559 & 5.5718 & 5.5718 & 8.5384 & & \\
\hline & 500 & Pres. $(5 \times 5)$ & 3.3461 & 7.7004 & 7.7004 & 16.2013 & 17.9808 & 24.1174 \\
\hline & & Pres. $(7 \times 7)$ & 3.3412 & 6.0691 & 6.0691 & 9.1488 & 11.1368 & 11.1426 \\
\hline & & Pres. $(9 \times 9)$ & 3.3411 & 5.9513 & 5.9513 & 8.8208 & 10.9377 & 10.9399 \\
\hline & & Pres. $(11 \times 11)$ & 3.3412 & 5.9445 & 5.9445 & 8.8028 & 10.8174 & 10.8209 \\
\hline & & Pres. $(13 \times 13)$ & 3.3410 & 5.9420 & 5.9420 & 8.7986 & 10.7885 & 10.7889 \\
\hline & & Pres. $(15 \times 15)$ & 3.3409 & 5.9404 & 5.9404 & 8.7965 & 10.7778 & 10.7781 \\
\hline & & Pres. $(17 \times 17)$ & 3.3408 & 5.9391 & 5.9391 & 8.7950 & 10.7708 & 10.7710 \\
\hline & & Pres. $(19 \times 19)$ & 3.3407 & 5.9380 & 5.9380 & 8.7937 & 10.7655 & 10.7656 \\
\hline & & Pres. $(23 \times 23)$ & 3.3406 & 5.9363 & 5.9363 & 8.7917 & 10.7576 & 10.7576 \\
\hline & & Ref. [1] & 3.3400 & 5.9287 & 5.9287 & 8.7775 & & \\
\hline & & Ref. [6] & 3.3398 & 5.9285 & 5.9285 & 8.7775 & & \\
\hline & & Ref. [7] & 3.3406 & 5.9285 & 5.9285 & 8.7754 & & \\
\hline \multirow[t]{24}{*}{0.1} & 200 & Pres. $(5 \times 5)$ & 2.8258 & 7.2782 & 7.2782 & 15.3872 & 16.8089 & 22.4779 \\
\hline & & Pres. $(7 \times 7)$ & 2.8204 & 5.6932 & 5.6932 & 8.6942 & 10.5696 & 10.5753 \\
\hline & & Pres. $(9 \times 9)$ & 2.8203 & 5.5745 & 5.5745 & 8.3761 & 10.3805 & 10.3827 \\
\hline & & Pres. $(11 \times 11)$ & 2.8204 & 5.5675 & 5.5675 & 8.3583 & 10.2646 & 10.2680 \\
\hline & & Pres. $(13 \times 13)$ & 2.8202 & 5.5649 & 5.5649 & 8.3542 & 10.2365 & 10.2369 \\
\hline & & Pres. $(15 \times 15)$ & 2.8200 & 5.5633 & 5.5633 & 8.3522 & 10.2261 & 10.2264 \\
\hline & & Pres. $(17 \times 17)$ & 2.8199 & 5.5620 & 5.5620 & 8.3507 & 10.2193 & 10.2195 \\
\hline & & Pres. $(19 \times 19)$ & 2.8198 & 5.5608 & 5.5608 & 8.3494 & 10.2141 & 10.2142 \\
\hline & & Pres. $(23 \times 23)$ & 2.8197 & 5.5591 & 5.5591 & 8.3475 & 10.2064 & 10.2065 \\
\hline & & Ref. [1] & 2.7842 & 5.3043 & 5.3043 & 7.7287 & & \\
\hline & & Ref. [6] & 2.7756 & 5.2954 & 5.2954 & 7.7279 & & \\
\hline & & Ref. [7] & 2.7902 & 5.3452 & 5.3452 & 7.8255 & & \\
\hline & 1000 & Pres. $(5 \times 5)$ & 4.0088 & 7.9497 & 7.9497 & 15.6297 & 18.2908 & 23.9416 \\
\hline & & Pres. $(7 \times 7)$ & 4.0044 & 6.3524 & 6.3524 & 9.1412 & 10.9246 & 10.9300 \\
\hline & & Pres. $(9 \times 9)$ & 4.0043 & 6.2421 & 6.2421 & 8.8244 & 10.7406 & 10.7428 \\
\hline & & Pres. $(11 \times 11)$ & 4.0043 & 6.2358 & 6.2358 & 8.8073 & 10.6279 & 10.6312 \\
\hline & & Pres. $(13 \times 13)$ & 4.0042 & 6.2335 & 6.2335 & 8.8033 & 10.6007 & 10.6011 \\
\hline & & Pres. $(15 \times 15)$ & 4.0041 & 6.2321 & 6.2321 & 8.8014 & 10.5907 & 10.5909 \\
\hline & & Pres. $(17 \times 17)$ & 4.0040 & 6.2309 & 6.2309 & 8.8000 & 10.5841 & 10.5842 \\
\hline & & Pres. $(19 \times 19)$ & 4.0039 & 6.2298 & 6.2298 & 8.7988 & 10.5791 & 10.5792 \\
\hline & & Pres. $(23 \times 23)$ & 4.0038 & 6.2283 & 6.2283 & 8.7969 & 10.5717 & 10.5717 \\
\hline & & Ref. [1] & 3.9805 & 6.0078 & 6.0078 & 8.2214 & & \\
\hline & & Ref. [6] & 3.9566 & 5.9757 & 5.9757 & 8.1954 & & \\
\hline & & Ref. [7] & 3.9844 & 6.0430 & 6.0430 & 8.3112 & & \\
\hline
\end{tabular}

Where $\varepsilon_{F}$ and $\sigma_{F}$ are regarded as strain and stress vectors associated with Pasternak foundation and are defines as follows,

$$
\begin{aligned}
\boldsymbol{\varepsilon}_{F} & =\left\{1 \frac{\partial}{\partial x} \frac{\partial}{\partial y}\right\}^{\mathrm{T}} w=\mathbf{L}_{f s} w \\
\boldsymbol{\sigma}_{F} & =\mathrm{D}_{F} \boldsymbol{\varepsilon}_{F}
\end{aligned}
$$


Table 5

Dimensionless parameter of natural frequencies, $\bar{\omega}=\frac{\omega a^{2}}{\pi^{2}} \sqrt{\frac{\rho h}{D}}$, of a square CCCC plate resting on Pasternak's type elastic foundation $\left(\mathrm{K}_{1}=\right.$ $1390.2, \mathrm{~K}_{2}=16683, \mathrm{~h} / \mathrm{a}=0.015$ and $\left.\nu=0.15\right)$

\begin{tabular}{|c|c|c|c|c|c|c|}
\hline \multirow[t]{2}{*}{ Method } & \multicolumn{6}{|c|}{ Mode number } \\
\hline & $1 \mathrm{st}$ & 2 nd & $3 \mathrm{rd}$ & 4th & 5 th & 6th \\
\hline Pres. $(5 \times 5)$ & 11.2731 & 487.5740 & 487.5740 & 691.8086 & 749.8748 & 773.9894 \\
\hline Pres. $(7 \times 7)$ & 8.4543 & 14.2932 & 14.2932 & 22.5495 & 25.5613 & 39.8698 \\
\hline Pres. $(9 \times 9)$ & 8.1614 & 12.9504 & 12.9504 & 17.2984 & 19.5261 & 19.8530 \\
\hline Pres. $(11 \times 11)$ & 8.1367 & 12.7800 & 12.7800 & 16.8966 & 19.2998 & 19.4499 \\
\hline Pres. $(13 \times 13)$ & 8.1281 & 12.7478 & 12.7478 & 16.7403 & 19.2333 & 19.2945 \\
\hline Pres. $(15 \times 15)$ & 8.1254 & 12.7336 & 12.7336 & 16.7012 & 19.2142 & 19.2536 \\
\hline Pres. $(17 \times 17)$ & 8.1240 & 12.7261 & 12.7261 & 16.6797 & 19.2071 & 19.2358 \\
\hline Pres. $(19 \times 19)$ & 8.1230 & 12.7214 & 12.7214 & 16.6700 & 19.2001 & 19.2236 \\
\hline Pres. $(23 \times 23)$ & 8.1214 & 12.7152 & 12.7152 & 16.6606 & 19.1867 & 19.2058 \\
\hline Ref. [2] & 8.1375 & 12.898 & 12.898 & 16.932 & & \\
\hline Ref. [6] & 8.1675 & 12.823 & 12.823 & 16.833 & & \\
\hline Ref. [7] & 8.1669 & 12.821 & 12.821 & 16.842 & & \\
\hline
\end{tabular}

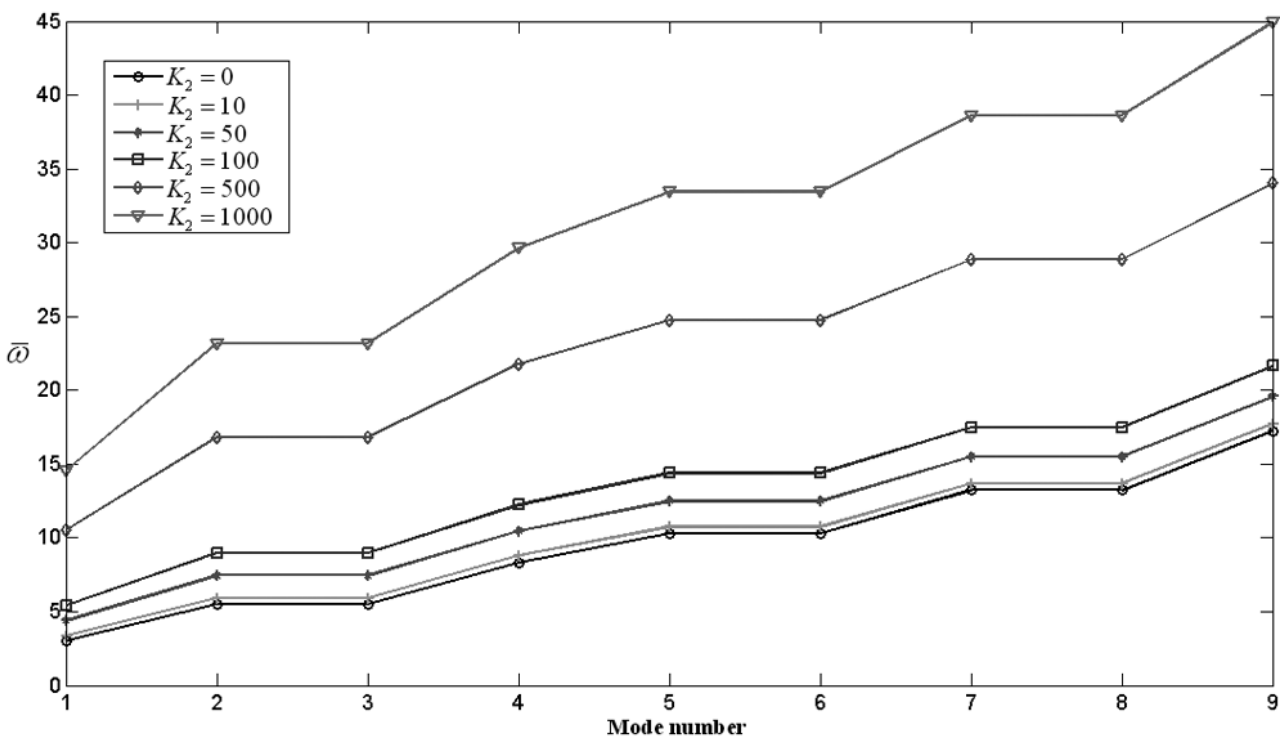

Fig. 5. Comparison of natural frequencies for Winkler and Pasternak's type of elastic foundation $\left(K_{1}=500, h / a=0.01\right.$ and number of nodes $19 \times 19)$.

where $\mathrm{D}_{F}$ is given by,

$$
\mathrm{D}_{F}=\left[\begin{array}{ccc}
k_{f} & 0 & 0 \\
0 & G_{f} & 0 \\
0 & 0 & G_{f}
\end{array}\right]
$$

\subsection{Approximation of field variables}

By using the MLS method the following approximation of deflection function in $\mathrm{z}$ direction is obtained:

$$
w^{h}(\mathbf{x})=\sum_{I=1}^{n} \phi_{I}(\mathbf{x}) w_{I}
$$


Table 6

Dimensionless parameter of natural frequencies, $\bar{\omega}=\frac{\omega a^{2}}{\pi^{2}} \sqrt{\frac{\rho h}{D}}$, of a square plate on Pasternak's type elastic foundation $\left(\mathrm{K}_{2}=10, \mathrm{~h} / \mathrm{a}=0.01\right.$ and number of nodes $19 \times 19)$

\begin{tabular}{|c|c|c|c|c|c|c|c|}
\hline \multirow[t]{2}{*}{ B.C. } & \multirow[t]{2}{*}{$\mathrm{K}_{1}$} & \multicolumn{6}{|c|}{ Mode number } \\
\hline & & $1 \mathrm{st}$ & $2 \mathrm{nd}$ & $3 \mathrm{rd}$ & 4th & 5 th & 6th \\
\hline SCSC & $\begin{array}{l}100 \\
500 \\
1000\end{array}$ & $\begin{array}{l}3.4527 \\
4.0033 \\
4.5999\end{array}$ & $\begin{array}{l}6.0949 \\
6.4228 \\
6.8105\end{array}$ & $\begin{array}{l}7.5255 \\
7.7934 \\
8.1159\end{array}$ & $\begin{array}{l}10.1074 \\
10.3084 \\
10.5543\end{array}$ & $\begin{array}{l}10.9209 \\
11.1072 \\
11.3357\end{array}$ & $\begin{array}{l}13.6416 \\
13.7912 \\
13.9759\end{array}$ \\
\hline SSCC & $\begin{array}{l}100 \\
500 \\
1000\end{array}$ & $\begin{array}{l}3.2945 \\
3.8677 \\
4.4823\end{array}$ & $\begin{array}{l}6.6644 \\
6.9656 \\
7.3246\end{array}$ & $\begin{array}{l}6.6846 \\
6.9849 \\
7.3430\end{array}$ & $\begin{array}{l}9.9326 \\
10.1370 \\
10.3870\end{array}$ & $\begin{array}{l}12.1658 \\
12.3332 \\
12.5394\end{array}$ & $\begin{array}{l}12.1805 \\
12.3478 \\
12.5537\end{array}$ \\
\hline SFSS & $\begin{array}{l}100 \\
500 \\
1000\end{array}$ & $\begin{array}{l}1.9313 \\
2.7992 \\
3.6011\end{array}$ & $\begin{array}{l}3.5429 \\
4.0814 \\
4.6679\end{array}$ & $\begin{array}{l}4.7833 \\
5.1947 \\
5.6671\end{array}$ & $\begin{array}{l}6.5913 \\
6.8956 \\
7.2580\end{array}$ & $\begin{array}{l}6.9730 \\
7.2613 \\
7.6063\end{array}$ & $\begin{array}{l}9.7393 \\
9.9478 \\
10.2023\end{array}$ \\
\hline FFSS & $\begin{array}{l}100 \\
500 \\
1000\end{array}$ & $\begin{array}{l}1.2994 \\
2.4072 \\
3.3057\end{array}$ & $\begin{array}{l}2.6769 \\
3.3572 \\
4.0500\end{array}$ & $\begin{array}{l}2.7733 \\
3.4346 \\
4.1144\end{array}$ & $\begin{array}{l}4.5871 \\
5.0146 \\
5.5024\end{array}$ & $\begin{array}{l}5.9674 \\
6.3019 \\
6.6966\end{array}$ & $\begin{array}{l}6.1585 \\
6.4831 \\
6.8674\end{array}$ \\
\hline FFSC & $\begin{array}{l}100 \\
500 \\
1000\end{array}$ & $\begin{array}{l}1.4006 \\
2.4633 \\
3.3467\end{array}$ & $\begin{array}{l}2.7949 \\
3.4521 \\
4.1290\end{array}$ & $\begin{array}{l}3.2738 \\
3.8501 \\
4.4671\end{array}$ & $\begin{array}{l}5.0515 \\
5.4427 \\
5.8952\end{array}$ & $\begin{array}{l}6.1053 \\
6.4326 \\
6.8197\end{array}$ & $\begin{array}{l}7.1681 \\
7.4489 \\
7.7856\end{array}$ \\
\hline FSFS & $\begin{array}{l}100 \\
500 \\
1000\end{array}$ & $\begin{array}{l}1.7330 \\
2.6663 \\
3.4988\end{array}$ & $\begin{array}{l}2.4271 \\
3.1617 \\
3.8895\end{array}$ & $\begin{array}{l}4.5545 \\
4.9848 \\
5.4753\end{array}$ & $\begin{array}{l}4.5559 \\
4.9861 \\
5.4765\end{array}$ & $\begin{array}{l}5.3624 \\
5.7323 \\
6.1636\end{array}$ & $\begin{array}{l}7.8203 \\
8.0784 \\
8.3899\end{array}$ \\
\hline FSFC & $\begin{array}{l}100 \\
500 \\
1000\end{array}$ & $\begin{array}{l}2.1435 \\
2.9497 \\
3.7193\end{array}$ & $\begin{array}{l}2.7784 \\
3.4387 \\
4.1179\end{array}$ & $\begin{array}{l}4.8181 \\
5.2267 \\
5.6964\end{array}$ & $\begin{array}{l}5.5511 \\
5.9093 \\
6.3286\end{array}$ & $\begin{array}{l}6.2747 \\
6.5937 \\
6.9719\end{array}$ & $\begin{array}{l}8.5774 \\
8.8133 \\
9.0997\end{array}$ \\
\hline FSFF & $\begin{array}{l}100 \\
500 \\
1000\end{array}$ & $\begin{array}{l}1.1427 \\
2.3264 \\
3.2473\end{array}$ & $\begin{array}{l}1.7063 \\
2.6490 \\
3.4856\end{array}$ & $\begin{array}{l}2.4654 \\
3.1912 \\
3.9135\end{array}$ & $\begin{array}{l}3.3679 \\
3.9303 \\
4.5364\end{array}$ & $\begin{array}{l}3.6260 \\
4.1536 \\
4.7311\end{array}$ & $\begin{array}{l}5.7051 \\
6.0541 \\
6.4640\end{array}$ \\
\hline SFSC & $\begin{array}{l}100 \\
500 \\
1000\end{array}$ & $\begin{array}{l}2.0138 \\
2.8568 \\
3.6460\end{array}$ & $\begin{array}{l}4.0416 \\
4.5211 \\
5.0568\end{array}$ & $\begin{array}{l}4.8327 \\
5.2402 \\
5.7088\end{array}$ & $\begin{array}{l}6.9808 \\
7.2688 \\
7.6135\end{array}$ & $\begin{array}{l}8.0060 \\
8.2583 \\
8.5632\end{array}$ & $\begin{array}{l}9.7713 \\
9.9791 \\
10.2328\end{array}$ \\
\hline $\mathrm{CCCC}$ & $\begin{array}{l}100 \\
500 \\
1000\end{array}$ & $\begin{array}{l}4.1067 \\
4.5793 \\
5.1090\end{array}$ & $\begin{array}{l}7.9246 \\
8.1795 \\
8.4873\end{array}$ & $\begin{array}{l}7.9246 \\
8.1795 \\
8.4873\end{array}$ & $\begin{array}{l}11.4696 \\
11.6471 \\
11.8652\end{array}$ & $\begin{array}{l}13.8906 \\
14.0375 \\
14.2190\end{array}$ & $\begin{array}{l}13.9520 \\
14.0983 \\
14.2790\end{array}$ \\
\hline CFCC & $\begin{array}{l}100 \\
500 \\
1000\end{array}$ & $\begin{array}{l}2.9256 \\
3.5588 \\
4.2187\end{array}$ & $\begin{array}{l}4.6645 \\
5.0856 \\
5.5672\end{array}$ & $\begin{array}{l}6.9083 \\
7.1993 \\
7.5472\end{array}$ & $\begin{array}{l}8.4202 \\
8.6605 \\
8.9517\end{array}$ & $\begin{array}{l}8.7004 \\
8.9332 \\
9.2158\end{array}$ & $\begin{array}{l}12.4004 \\
12.5648 \\
12.7672\end{array}$ \\
\hline CFSC & $\begin{array}{l}100 \\
500 \\
1000\end{array}$ & $\begin{array}{l}2.3923 \\
3.1351 \\
3.8680\end{array}$ & $\begin{array}{l}4.3066 \\
4.7594 \\
5.2710\end{array}$ & $\begin{array}{l}5.7953 \\
6.1393 \\
6.5438\end{array}$ & $\begin{array}{l}7.7673 \\
8.0271 \\
8.3405\end{array}$ & $\begin{array}{l}8.1903 \\
8.4371 \\
8.7358\end{array}$ & $\begin{array}{l}11.2920 \\
11.4723 \\
11.6937\end{array}$ \\
\hline $\mathrm{CFCF}$ & $\begin{array}{l}100 \\
500 \\
1000\end{array}$ & $\begin{array}{l}2.7181 \\
3.3903 \\
4.0775\end{array}$ & $\begin{array}{l}3.2639 \\
3.8417 \\
4.4599\end{array}$ & $\begin{array}{l}5.1602 \\
5.5437 \\
5.9886\end{array}$ & $\begin{array}{l}6.6934 \\
6.9933 \\
7.3510\end{array}$ & $\begin{array}{l}7.3379 \\
7.6124 \\
7.9422\end{array}$ & $\begin{array}{l}8.8761 \\
9.1043 \\
9.3817\end{array}$ \\
\hline FFCC & $\begin{array}{l}100 \\
500 \\
1000\end{array}$ & $\begin{array}{l}1.4975 \\
2.5197 \\
3.3884\end{array}$ & $\begin{array}{l}3.2617 \\
3.8398 \\
4.4582\end{array}$ & $\begin{array}{l}3.4074 \\
3.9643 \\
4.5659\end{array}$ & $\begin{array}{l}5.4896 \\
5.8516 \\
6.2747\end{array}$ & $\begin{array}{l}7.0872 \\
7.3710 \\
7.7112\end{array}$ & $\begin{array}{l}7.3222 \\
7.5972 \\
7.9276\end{array}$ \\
\hline FFCF & $\begin{array}{l}100 \\
500 \\
1000\end{array}$ & $\begin{array}{l}1.2460 \\
2.3788 \\
3.2851\end{array}$ & $\begin{array}{l}1.8048 \\
2.7135 \\
3.5349\end{array}$ & $\begin{array}{l}3.0164 \\
3.6337 \\
4.2820\end{array}$ & $\begin{array}{l}3.7057 \\
4.2234 \\
4.7925\end{array}$ & $\begin{array}{l}3.8825 \\
4.3794 \\
4.9306\end{array}$ & $\begin{array}{l}6.2183 \\
6.5400 \\
6.9211\end{array}$ \\
\hline CCSC & $\begin{array}{l}100 \\
500 \\
1000\end{array}$ & $\begin{array}{l}3.7221 \\
4.2379 \\
4.8054\end{array}$ & $\begin{array}{l}6.9323 \\
7.2223 \\
7.5691\end{array}$ & $\begin{array}{l}7.6980 \\
7.9601 \\
8.2761\end{array}$ & $\begin{array}{l}10.7266 \\
10.9162 \\
11.1487\end{array}$ & $\begin{array}{l}12.3447 \\
12.5097 \\
12.7131\end{array}$ & $\begin{array}{l}13.7673 \\
13.9155 \\
14.0985\end{array}$ \\
\hline CSSS & $\begin{array}{l}100 \\
500 \\
1000\end{array}$ & $\begin{array}{l}2.9899 \\
3.6118 \\
4.2635\end{array}$ & $\begin{array}{l}5.8036 \\
6.1470 \\
6.5511\end{array}$ & $\begin{array}{l}6.4784 \\
6.7878 \\
7.1558\end{array}$ & $\begin{array}{l}9.2667 \\
9.4856 \\
9.7522\end{array}$ & $\begin{array}{l}10.7290 \\
10.9185 \\
11.1509\end{array}$ & $\begin{array}{l}12.0336 \\
12.2028 \\
12.4112\end{array}$ \\
\hline
\end{tabular}


Table 6, continued

\begin{tabular}{llllcccc}
\hline B.C. & $\mathrm{K}_{1}$ & \multicolumn{7}{c}{ Mode number } \\
\cline { 3 - 8 } & & 1st & 2nd & 3rd & 4th & 5th & 6th \\
\hline FFFF & 100 & 1.0132 & 1.4870 & 1.4870 & 2.2656 & 3.1826 & 3.4509 \\
& 500 & 2.2656 & 2.5134 & 2.5134 & 3.0395 & 3.7727 & 4.0016 \\
& 1000 & 3.2041 & 3.3837 & 3.3837 & 3.7908 & 4.4005 & 4.5983 \\
\hline
\end{tabular}

\subsection{Imposing essential boundary conditions}

The essential boundary conditions in the element free Galerkin method cannot be easily and directly imposed because moving least square approximation does not satisfy the Kronecker delta function property, [22]. Various techniques have been proposed for imposing the essential boundary conditions in the EFG method, such as Lagrange multipliers [21], penalty method [23], coupling with FEM [24], and coupling with boundary element method [25]. In this paper, a Penalty method is used for enforcing the essential boundary condition in the EFG method.

In the absence of body forces and assumed prescribed tractions, Lagrangian of free vibration of a thin plate resting on elastic foundation can be written as follows

$$
L=T_{P}-U_{p}-U_{F}+\int_{\Gamma_{u}} \frac{1}{2}(\tilde{\mathbf{u}}-\overline{\mathbf{u}})^{\mathrm{T}} \boldsymbol{\alpha}(\tilde{\mathbf{u}}-\overline{\mathbf{u}}) \mathrm{d} \Gamma,
$$

where $\boldsymbol{\alpha}$ is a diagonal matrix of penalty coefficients, $\overline{\mathbf{u}}$ is prescribed displacement and defines as [26],

$$
\tilde{\mathbf{u}}=\mathbf{L}_{b} w
$$

where $\mathbf{L}_{b}$ is a vector of differential operators. Proper choice of penalty coefficients is very important on accuracy of solution. From literature review [26], usually large numbers of order $1 \times 10^{4-13} \times \max$ (diagonals in stiffness matrix) can be chosen. A plate with general boundary condition is considered (Fig. 3). For clamped boundary condition $\mathbf{L}_{b}$ is defined as,

$$
\mathbf{L}_{b}=\left\{1 \frac{\partial}{\partial n}\right\}^{\mathrm{T}}
$$

where $n$ denotes outward normal direction on boundary of the plate. For simply supported boundary condition $\mathbf{L}_{b}$ is defined as,

$$
\mathbf{L}_{b}=\left\{\begin{array}{ll}
1 & 0
\end{array}\right\}^{\mathrm{T}}
$$

\subsection{Derivation of stiffness and mass matrices for free vibration analysis}

The dynamical equation of a plate resting on elastic foundation can be derived according to the Hamilton's variational principle,

$$
\delta \int_{t_{1}}^{t_{2}} L \mathrm{~d} t=0
$$

where $L$ is the Lagrangian function of system. By substituting Eqs (37), (38) and (40) into Eq. (45) and replacing results in Eq. (49), following variational form is found:

$$
\int_{S} \delta \varepsilon_{P}^{\mathrm{T}} \boldsymbol{\sigma}_{P} \mathrm{~d} S+\int_{S} \delta \varepsilon_{F}^{\mathrm{T}} \boldsymbol{\sigma}_{F} \mathrm{~d} S+\int_{V} \rho \delta \mathbf{u}^{\mathrm{T}} \ddot{\mathbf{u}} \mathrm{d} V-\delta \int_{\Gamma_{u}} \frac{1}{2}(\tilde{\mathbf{u}}-\overline{\mathbf{u}})^{\mathrm{T}} \boldsymbol{\alpha}(\tilde{\mathbf{u}}-\overline{\mathbf{u}}) d \Gamma=0
$$


Table 7

Dimensionless parameter of natural frequencies, $\bar{\omega}=\frac{\omega a^{2}}{\pi^{2}} \sqrt{\frac{\rho h}{D}}$, of a square plate on Pasternak's type elastic foundation $\left(\mathrm{K}_{2}=50, \mathrm{~h} / \mathrm{a}=0.01\right.$ and number of nodes $19 \times 19$ )

\begin{tabular}{|c|c|c|c|c|c|c|c|}
\hline \multirow[t]{2}{*}{ B.C } & \multirow[t]{2}{*}{$\mathrm{K}_{1}$} & \multicolumn{6}{|c|}{ Mode number } \\
\hline & & $1 \mathrm{st}$ & 2nd & 3rd & 4th & 5 th & 6th \\
\hline SSSS & $\begin{array}{l}100 \\
500 \\
1000\end{array}$ & $\begin{array}{l}3.8935 \\
4.3892 \\
4.9393\end{array}$ & $\begin{array}{l}7.1703 \\
7.4510 \\
7.7877\end{array}$ & $\begin{array}{l}7.1703 \\
7.4510 \\
7.7877\end{array}$ & $\begin{array}{l}10.2777 \\
10.4754 \\
10.7175\end{array}$ & $\begin{array}{l}12.3409 \\
12.5060 \\
12.7094\end{array}$ & $\begin{array}{l}12.3410 \\
12.5062 \\
12.7096\end{array}$ \\
\hline SCSC & $\begin{array}{l}100 \\
500 \\
1000\end{array}$ & $\begin{array}{l}4.5622 \\
4.9919 \\
5.4819\end{array}$ & $\begin{array}{l}7.6189 \\
7.8837 \\
8.2027\end{array}$ & $\begin{array}{l}8.9035 \\
9.1311 \\
9.4078\end{array}$ & $\begin{array}{l}11.6903 \\
11.8646 \\
12.0788\end{array}$ & $\begin{array}{l}12.6604 \\
12.8214 \\
13.0199\end{array}$ & $\begin{array}{l}15.1832 \\
15.3177 \\
15.4842\end{array}$ \\
\hline SSCC & $\begin{array}{l}100 \\
500 \\
1000\end{array}$ & $\begin{array}{l}4.4560 \\
4.8950 \\
5.3938\end{array}$ & $\begin{array}{l}8.1437 \\
8.3920 \\
8.6923\end{array}$ & $\begin{array}{l}8.1547 \\
8.4026 \\
8.7026\end{array}$ & $\begin{array}{l}11.5434 \\
11.7198 \\
11.9366\end{array}$ & $\begin{array}{l}13.8154 \\
13.9631 \\
14.1456\end{array}$ & $\begin{array}{l}13.8260 \\
13.9736 \\
14.1559\end{array}$ \\
\hline SFSS & $\begin{array}{l}100 \\
500 \\
1000\end{array}$ & $\begin{array}{l}2.9739 \\
3.5987 \\
4.2524\end{array}$ & $\begin{array}{l}5.1403 \\
5.5252 \\
5.9716\end{array}$ & $\begin{array}{l}6.3433 \\
6.6590 \\
7.0337\end{array}$ & $\begin{array}{l}8.3280 \\
8.5709 \\
8.8651\end{array}$ & $\begin{array}{l}8.9734 \\
9.1993 \\
9.4740\end{array}$ & $\begin{array}{l}11.5147 \\
11.6915 \\
11.9088\end{array}$ \\
\hline FFSS & $\begin{array}{l}100 \\
500 \\
1000\end{array}$ & $\begin{array}{l}1.9380 \\
2.8039 \\
3.6048\end{array}$ & $\begin{array}{l}4.2715 \\
4.7277 \\
5.2424\end{array}$ & $\begin{array}{l}4.2980 \\
4.7517 \\
5.2641\end{array}$ & $\begin{array}{l}6.3562 \\
6.6713 \\
7.0454\end{array}$ & $\begin{array}{l}8.0792 \\
8.3293 \\
8.6317\end{array}$ & $\begin{array}{l}8.1668 \\
8.4143 \\
8.7138\end{array}$ \\
\hline FFSC & $\begin{array}{l}100 \\
500 \\
1000\end{array}$ & $\begin{array}{l}2.0550 \\
2.8860 \\
3.6690\end{array}$ & $\begin{array}{l}4.3485 \\
4.7974 \\
5.3054\end{array}$ & $\begin{array}{l}4.8120 \\
5.2211 \\
5.6914\end{array}$ & $\begin{array}{l}6.7846 \\
7.0807 \\
7.4342\end{array}$ & $\begin{array}{l}8.1631 \\
8.4107 \\
8.7103\end{array}$ & $\begin{array}{l}9.1225 \\
9.3447 \\
9.6153\end{array}$ \\
\hline FSFS & $\begin{array}{l}100 \\
500 \\
1000\end{array}$ & $\begin{array}{l}2.6590 \\
3.3431 \\
4.0384\end{array}$ & $\begin{array}{l}3.7868 \\
4.2949 \\
4.0384\end{array}$ & $\begin{array}{l}6.0872 \\
6.4156 \\
6.8037\end{array}$ & $\begin{array}{l}6.5713 \\
6.8765 \\
7.2400\end{array}$ & $\begin{array}{l}7.0341 \\
7.3201 \\
7.6626\end{array}$ & $\begin{array}{l}9.7677 \\
9.9755 \\
10.2294\end{array}$ \\
\hline FSFC & $\begin{array}{l}100 \\
500 \\
1000\end{array}$ & $\begin{array}{l}3.0310 \\
3.6460 \\
4.2925\end{array}$ & $\begin{array}{l}4.0790 \\
4.5545 \\
5.0868\end{array}$ & $\begin{array}{l}6.7819 \\
7.0781 \\
7.4317\end{array}$ & $\begin{array}{l}6.9614 \\
7.2503 \\
7.5959\end{array}$ & $\begin{array}{l}7.8353 \\
8.0930 \\
8.4040\end{array}$ & $\begin{array}{l}10.4368 \\
10.6316 \\
10.8701\end{array}$ \\
\hline FSFF & $\begin{array}{l}100 \\
500 \\
1000\end{array}$ & $\begin{array}{l}1.5287 \\
2.5384 \\
3.4024\end{array}$ & $\begin{array}{l}2.8786 \\
3.5203 \\
4.1863\end{array}$ & $\begin{array}{l}4.0043 \\
4.4878 \\
5.0271\end{array}$ & $\begin{array}{l}5.0427 \\
5.4345 \\
5.8877\end{array}$ & $\begin{array}{l}5.7190 \\
6.0673 \\
6.4763\end{array}$ & $\begin{array}{l}7.7912 \\
8.0503 \\
8.3629\end{array}$ \\
\hline SFSC & $\begin{array}{l}100 \\
500 \\
1000\end{array}$ & $\begin{array}{l}3.0571 \\
3.6676 \\
4.3109\end{array}$ & $\begin{array}{l}5.6181 \\
5.9722 \\
6.3874\end{array}$ & $\begin{array}{l}6.3909 \\
6.7044 \\
7.0767\end{array}$ & $\begin{array}{l}8.6962 \\
8.9291 \\
9.2119\end{array}$ & $\begin{array}{l}9.9272 \\
10.1318 \\
10.3819\end{array}$ & $\begin{array}{l}11.5463 \\
11.7226 \\
11.9394\end{array}$ \\
\hline $\mathrm{CCCC}$ & $\begin{array}{l}100 \\
500 \\
1000\end{array}$ & $\begin{array}{l}5.1501 \\
5.5344 \\
5.9801\end{array}$ & $\begin{array}{l}9.2762 \\
9.4949 \\
9.7613\end{array}$ & $\begin{array}{l}9.2762 \\
9.4949 \\
9.7613\end{array}$ & $\begin{array}{l}12.9650 \\
13.1223 \\
13.3163\end{array}$ & $\begin{array}{l}15.4286 \\
15.5610 \\
15.7249\end{array}$ & $\begin{array}{l}15.4739 \\
15.6059 \\
15.7694\end{array}$ \\
\hline CFCC & $\begin{array}{l}100 \\
500 \\
1000\end{array}$ & $\begin{array}{l}3.8338 \\
4.3364 \\
4.8925\end{array}$ & $\begin{array}{l}6.1366 \\
6.4624 \\
6.8479\end{array}$ & $\begin{array}{l}8.2287 \\
8.4745 \\
8.7720\end{array}$ & $\begin{array}{l}10.2369 \\
10.4355 \\
10.6784\end{array}$ & $\begin{array}{l}10.2900 \\
10.4875 \\
10.7292\end{array}$ & $\begin{array}{l}14.1867 \\
14.3306 \\
14.5084\end{array}$ \\
\hline CFSC & $\begin{array}{l}100 \\
500 \\
1000\end{array}$ & $\begin{array}{l}3.3938 \\
3.9527 \\
4.5559\end{array}$ & $\begin{array}{l}5.8436 \\
6.1849 \\
6.5866\end{array}$ & $\begin{array}{l}7.2391 \\
7.5172 \\
7.8511\end{array}$ & $\begin{array}{l}9.4024 \\
9.6182 \\
9.8813\end{array}$ & $\begin{array}{l}10.0895 \\
10.2909 \\
10.5372\end{array}$ & $\begin{array}{l}12.9407 \\
13.0982 \\
13.2926\end{array}$ \\
\hline $\mathrm{CFCF}$ & $\begin{array}{l}100 \\
500 \\
1000\end{array}$ & $\begin{array}{l}3.5109 \\
4.0537 \\
4.6438\end{array}$ & $\begin{array}{l}4.4624 \\
4.9009 \\
5.3991\end{array}$ & $\begin{array}{l}7.0497 \\
7.3351 \\
7.6768\end{array}$ & $\begin{array}{l}7.9770 \\
8.2303 \\
8.5363\end{array}$ & $\begin{array}{l}8.7751 \\
9.0060 \\
9.2865\end{array}$ & $\begin{array}{l}11.2077 \\
11.3893 \\
11.6122\end{array}$ \\
\hline FFCC & $\begin{array}{l}100 \\
500 \\
1000\end{array}$ & $\begin{array}{l}2.1659 \\
2.9661 \\
3.7323\end{array}$ & $\begin{array}{l}4.8504 \\
5.2566 \\
5.7239\end{array}$ & $\begin{array}{l}4.8892 \\
5.2924 \\
5.7569\end{array}$ & $\begin{array}{l}7.1927 \\
7.4726 \\
7.8083\end{array}$ & $\begin{array}{l}9.0988 \\
9.3215 \\
9.5927\end{array}$ & $\begin{array}{l}9.2193 \\
9.4393 \\
9.7072\end{array}$ \\
\hline FFCF & $\begin{array}{l}100 \\
500 \\
1000\end{array}$ & $\begin{array}{l}1.6709 \\
2.6265 \\
3.4686\end{array}$ & $\begin{array}{l}2.9646 \\
3.5909 \\
4.2458\end{array}$ & $\begin{array}{l}4.5505 \\
4.9812 \\
5.4721\end{array}$ & $\begin{array}{l}5.5258 \\
5.8856 \\
6.3065\end{array}$ & $\begin{array}{l}5.7739 \\
6.1190 \\
6.5248\end{array}$ & $\begin{array}{l}8.2012 \\
8.4477 \\
8.7461\end{array}$ \\
\hline CCSC & $\begin{array}{l}100 \\
500 \\
1000\end{array}$ & $\begin{array}{l}4.8151 \\
5.2240 \\
5.6941\end{array}$ & $\begin{array}{l}8.3789 \\
8.6204 \\
8.9130\end{array}$ & $\begin{array}{l}9.0677 \\
9.2913 \\
9.5634\end{array}$ & $\begin{array}{l}12.2733 \\
12.4393 \\
12.6438\end{array}$ & $\begin{array}{l}13.9808 \\
14.1268 \\
14.3072\end{array}$ & $\begin{array}{l}15.3046 \\
15.4380 \\
15.6032\end{array}$ \\
\hline
\end{tabular}


Table 7, continued

\begin{tabular}{llllllll}
\hline B.C & $\mathrm{K}_{1}$ & \multicolumn{7}{c}{ Mode number } \\
\cline { 3 - 7 } & & 1st & 2nd & 3rd & 4th & 5th & 6th \\
\hline CSSS & 100 & 4.1831 & 7.3679 & 7.9683 & 10.9262 & 12.4853 & 13.6884 \\
& 500 & 4.6480 & 7.6414 & 8.2218 & 11.1124 & 12.6486 & 13.8374 \\
& 1000 & 5.1707 & 7.9701 & 8.5281 & 11.3409 & 12.8497 & 14.0215 \\
FFFF & 100 & 1.0132 & 2.5610 & 2.5610 & 3.7055 & 5.3937 & 5.4901 \\
& 500 & 2.2656 & 3.2657 & 3.2657 & 4.2233 & 5.7617 & 5.8520 \\
& 1000 & 3.2041 & 3.9746 & 3.9746 & 4.7925 & 6.1910 & 6.2752 \\
\hline
\end{tabular}

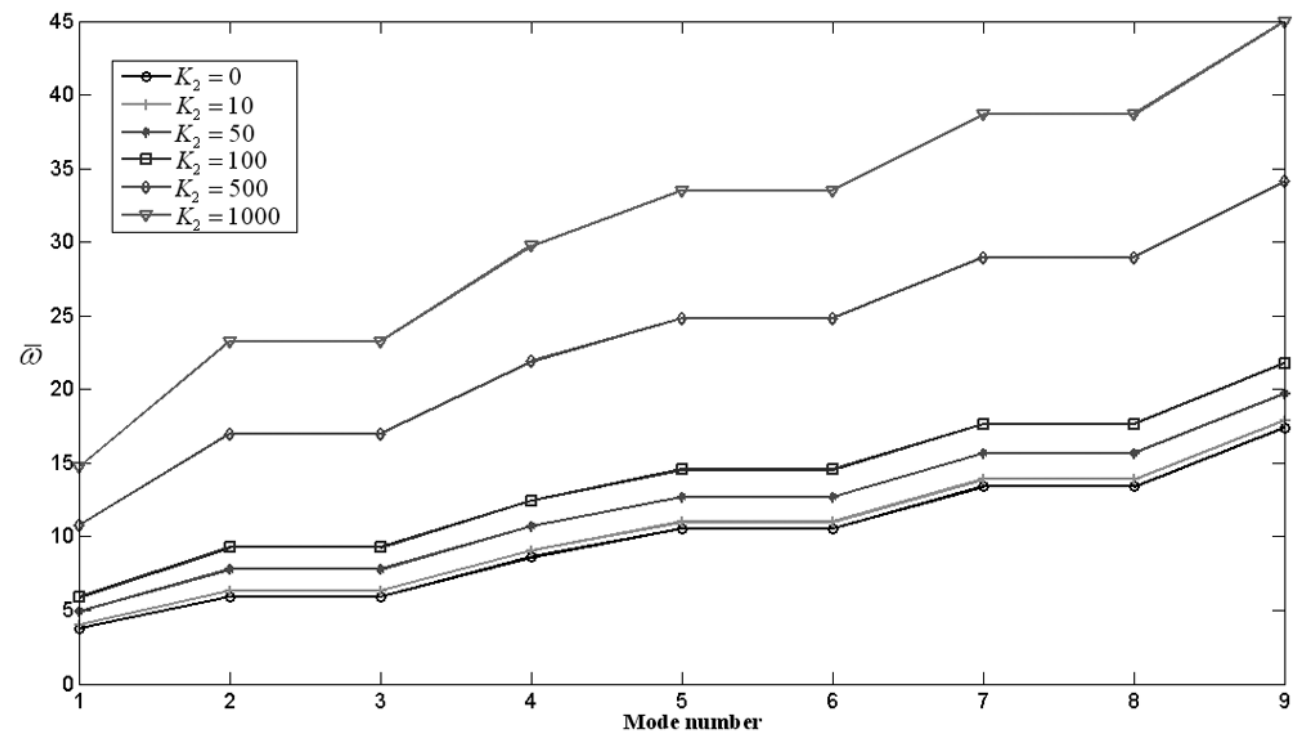

Fig. 6. Comparison of natural frequencies for Winkler and Pasternak's type of elastic foundation $\left(K_{1}=1000, h / a=0.01\right.$ and number of nodes $19 \times 19)$.

After substituting Eqs (31), (32), (34), (41), (42) and (46) into Eq. (50), it can be rewritten as:

$$
\begin{gathered}
\int_{S} \delta\left(\mathbf{L}_{p s} w\right)^{\mathrm{T}} \mathbf{D}\left(\mathbf{L}_{p s} w\right) \mathrm{d} S+\int_{S} \delta\left(\mathbf{L}_{f s} w\right)^{\mathrm{T}} \mathbf{D}_{F}\left(\mathbf{L}_{f s} w\right) \mathrm{d} S+\int_{V} \rho \delta\left(\mathbf{L}_{u} w\right)^{\mathrm{T}} \mathbf{L}_{u} \ddot{w} \mathrm{~d} V \\
-\frac{1}{2} \int_{\Gamma_{u}} \delta\left(\mathbf{L}_{b} w-\overline{\mathbf{u}}\right)^{\mathrm{T}} \boldsymbol{\alpha}\left(\mathbf{L}_{b} w-\overline{\mathbf{u}}\right) \mathrm{d} \Gamma=0
\end{gathered}
$$

By substituting the approximated deflection function $w^{h}(\mathbf{x})$ Eq. (44) into Eq. (51), the discrete dynamical equations for free vibration analysis of plates resting on elastic foundation is deduced as:

$$
\mathbf{M} \ddot{\mathbf{U}}+\left(\mathbf{K}_{P}+\mathbf{K}_{F}+\tilde{\mathbf{K}}\right) \mathbf{U}=0
$$

where $\mathbf{U}$ is the vector of deflection of all nodes, and is defined by

$$
\mathbf{U}=\left\{w_{1}, w_{2}, \ldots, w_{n_{t}}\right\}^{\mathrm{T}}
$$

Where $n_{t}$ is the total number of nodes in the entire domain of the plate. The notations of $\mathbf{K}_{P}, \mathbf{K}_{F}, \tilde{\mathbf{K}}$ and $\mathbf{M}$ denote the global plate stiffness matrix, the global foundation stiffness matrix, the global penalty matrix and global mass matrix which are given by:

$$
\mathbf{K}_{P}=\int_{S} \mathbf{B}_{I}^{\mathrm{T}} \mathbf{D} \mathbf{B}_{J} \mathrm{~d} S,
$$


Table 8

Dimensionless parameter of natural frequencies, $\bar{\omega}=\frac{\omega a^{2}}{\pi^{2}} \sqrt{\frac{\rho h}{D}}$, of a square plate on Pasternak's type elastic foundation $\left(\mathrm{K}_{2}=100, \mathrm{~h} / \mathrm{a}=0.01\right.$ and number of nodes $19 \times 19)$

\begin{tabular}{|c|c|c|c|c|c|c|c|}
\hline \multirow[t]{2}{*}{ B.C } & \multirow[t]{2}{*}{$\mathrm{K}_{1}$} & \multicolumn{6}{|c|}{ Mode number } \\
\hline & & $1 \mathrm{st}$ & $2 \mathrm{nd}$ & $3 \mathrm{rd}$ & 4th & 5 th & 6 th \\
\hline SSSS & $\begin{array}{l}100 \\
500 \\
1000\end{array}$ & $\begin{array}{l}5.0289 \\
5.4217 \\
5.8760\end{array}$ & $\begin{array}{l}8.7599 \\
8.9911 \\
9.2720\end{array}$ & $\begin{array}{l}8.7599 \\
8.9911 \\
9.2720\end{array}$ & $\begin{array}{l}12.0887 \\
12.2573 \\
12.4648\end{array}$ & $\begin{array}{l}14.2454 \\
14.3886 \\
14.5658\end{array}$ & $\begin{array}{l}14.2455 \\
14.3887 \\
14.5659\end{array}$ \\
\hline SCSC & $\begin{array}{l}100 \\
500 \\
1000\end{array}$ & $\begin{array}{l}5.6376 \\
5.9907 \\
6.4047\end{array}$ & $\begin{array}{l}9.1698 \\
9.3910 \\
9.6603\end{array}$ & $\begin{array}{l}10.3581 \\
10.5544 \\
10.7947\end{array}$ & $\begin{array}{l}13.4012 \\
13.5535 \\
13.7414\end{array}$ & $\begin{array}{l}14.5434 \\
14.6838 \\
14.8574\end{array}$ & $\begin{array}{l}16.9068 \\
17.0277 \\
17.1776\end{array}$ \\
\hline SSCC & $\begin{array}{l}100 \\
500 \\
1000\end{array}$ & $\begin{array}{l}5.5585 \\
5.9163 \\
6.3352\end{array}$ & $\begin{array}{l}9.6676 \\
9.8776 \\
10.1340\end{array}$ & $\begin{array}{l}9.6740 \\
9.8839 \\
10.1401\end{array}$ & $\begin{array}{l}13.2766 \\
13.4303 \\
13.6199\end{array}$ & $\begin{array}{l}15.6292 \\
15.7599 \\
15.9217\end{array}$ & $\begin{array}{l}15.6370 \\
15.7676 \\
15.9294\end{array}$ \\
\hline SFSS & $\begin{array}{l}100 \\
500 \\
1000\end{array}$ & $\begin{array}{l}3.8983 \\
4.3934 \\
4.9432\end{array}$ & $\begin{array}{l}6.5779 \\
6.8829 \\
7.2461\end{array}$ & $\begin{array}{l}7.8644 \\
8.1212 \\
8.4312\end{array}$ & $\begin{array}{l}10.0715 \\
10.2732 \\
10.5199\end{array}$ & $\begin{array}{l}10.9149 \\
11.1013 \\
11.3300\end{array}$ & $\begin{array}{l}13.4029 \\
13.5551 \\
13.7430\end{array}$ \\
\hline FFSS & $\begin{array}{l}100 \\
500 \\
1000\end{array}$ & $\begin{array}{l}2.5106 \\
3.2263 \\
3.9423\end{array}$ & $\begin{array}{l}5.5992 \\
5.9545 \\
6.3709\end{array}$ & $\begin{array}{l}5.6107 \\
5.9653 \\
6.3810\end{array}$ & $\begin{array}{l}7.9947 \\
8.2474 \\
8.5528\end{array}$ & $\begin{array}{l}10.0262 \\
10.2288 \\
10.4766\end{array}$ & $\begin{array}{l}10.0714 \\
10.2731 \\
10.5198\end{array}$ \\
\hline FFSC & $\begin{array}{l}100 \\
500 \\
1000\end{array}$ & $\begin{array}{l}2.6297 \\
3.3199 \\
4.0192\end{array}$ & $\begin{array}{l}5.6653 \\
6.0167 \\
6.4290\end{array}$ & $\begin{array}{l}6.1227 \\
6.4493 \\
6.8355\end{array}$ & $\begin{array}{l}8.4042 \\
8.6449 \\
8.9368\end{array}$ & $\begin{array}{l}10.0874 \\
10.2888 \\
10.5352\end{array}$ & $\begin{array}{l}11.0035 \\
11.1884 \\
11.4154\end{array}$ \\
\hline FSFS & $\begin{array}{l}100 \\
500 \\
1000\end{array}$ & $\begin{array}{l}3.4845 \\
4.0309 \\
4.6239\end{array}$ & $\begin{array}{l}4.9657 \\
5.3632 \\
5.8220\end{array}$ & $\begin{array}{l}7.5744 \\
7.8407 \\
8.1614\end{array}$ & $\begin{array}{l}8.3694 \\
8.6111 \\
8.9041\end{array}$ & $\begin{array}{l}8.6680 \\
8.9017 \\
9.1853\end{array}$ & $\begin{array}{l}11.7228 \\
11.8966 \\
12.1102\end{array}$ \\
\hline FSFC & $\begin{array}{l}100 \\
500 \\
1000\end{array}$ & $\begin{array}{l}3.8419 \\
4.3436 \\
4.8989\end{array}$ & $\begin{array}{l}5.2369 \\
5.6152 \\
6.0550\end{array}$ & $\begin{array}{l}8.3833 \\
8.6247 \\
8.9172\end{array}$ & $\begin{array}{l}8.5583 \\
8.7948 \\
9.0819\end{array}$ & $\begin{array}{l}9.4074 \\
9.6231 \\
9.8861\end{array}$ & $\begin{array}{l}12.3379 \\
12.5031 \\
12.7066\end{array}$ \\
\hline FSFF & $\begin{array}{l}100 \\
500 \\
1000\end{array}$ & $\begin{array}{l}1.8997 \\
2.7776 \\
3.5844\end{array}$ & $\begin{array}{l}3.8433 \\
4.3447 \\
4.8999\end{array}$ & $\begin{array}{l}5.2736 \\
5.6494 \\
6.0867\end{array}$ & $\begin{array}{l}6.5140 \\
6.8218 \\
7.1881\end{array}$ & $\begin{array}{l}7.4703 \\
7.7402 \\
8.0649\end{array}$ & $\begin{array}{l}9.7105 \\
9.9196 \\
10.1749\end{array}$ \\
\hline SFSC & $\begin{array}{l}100 \\
500 \\
1000\end{array}$ & $\begin{array}{l}3.9796 \\
4.4657 \\
5.0075\end{array}$ & $\begin{array}{l}7.0432 \\
7.3288 \\
7.6710\end{array}$ & $\begin{array}{l}7.9106 \\
8.1660 \\
8.4743\end{array}$ & $\begin{array}{l}10.4253 \\
10.6203 \\
10.8592\end{array}$ & $\begin{array}{l}11.8284 \\
12.0006 \\
12.2124\end{array}$ & $\begin{array}{l}13.4341 \\
13.5859 \\
13.7734\end{array}$ \\
\hline $\mathrm{CCCC}$ & $\begin{array}{l}100 \\
500 \\
1000\end{array}$ & $\begin{array}{l}6.1900 \\
6.5132 \\
6.8959\end{array}$ & $\begin{array}{l}10.7124 \\
10.9023 \\
11.1351\end{array}$ & $\begin{array}{l}10.7124 \\
10.9023 \\
11.1351\end{array}$ & $\begin{array}{l}14.6078 \\
14.7476 \\
14.9205\end{array}$ & $\begin{array}{l}17.1481 \\
17.2673 \\
17.4152\end{array}$ & $\begin{array}{l}17.1820 \\
17.3010 \\
17.4486\end{array}$ \\
\hline CFCC & $\begin{array}{l}100 \\
500 \\
1000\end{array}$ & $\begin{array}{l}4.6985 \\
5.1168 \\
5.5959\end{array}$ & $\begin{array}{l}7.5146 \\
7.7829 \\
8.1059\end{array}$ & $\begin{array}{l}9.6048 \\
9.8161 \\
10.0741\end{array}$ & $\begin{array}{l}11.8496 \\
12.0216 \\
12.2331\end{array}$ & $\begin{array}{l}12.1599 \\
12.3275 \\
12.5338\end{array}$ & $\begin{array}{l}16.1052 \\
16.2320 \\
16.3893\end{array}$ \\
\hline CFSC & $\begin{array}{l}100 \\
500 \\
1000\end{array}$ & $\begin{array}{l}4.3011 \\
4.7545 \\
5.2667\end{array}$ & $\begin{array}{l}7.2528 \\
7.5305 \\
7.8638\end{array}$ & $\begin{array}{l}8.6962 \\
8.9291 \\
9.2119\end{array}$ & $\begin{array}{l}11.0821 \\
11.2657 \\
11.4912\end{array}$ & $\begin{array}{l}11.9783 \\
12.1484 \\
12.3577\end{array}$ & $\begin{array}{l}14.7354 \\
14.8740 \\
15.0454\end{array}$ \\
\hline $\mathrm{CFCF}$ & $\begin{array}{l}100 \\
500 \\
1000\end{array}$ & $\begin{array}{l}4.2778 \\
4.7335 \\
5.2477\end{array}$ & $\begin{array}{l}5.5757 \\
5.9324 \\
6.3503\end{array}$ & $\begin{array}{l}8.7914 \\
9.0218 \\
9.3018\end{array}$ & $\begin{array}{l}9.3159 \\
9.5336 \\
9.7990\end{array}$ & $\begin{array}{l}10.2668 \\
10.4648 \\
10.7071\end{array}$ & $\begin{array}{l}13.0550 \\
13.2112 \\
13.4039\end{array}$ \\
\hline FFCC & $\begin{array}{l}100 \\
500 \\
1000\end{array}$ & $\begin{array}{l}2.7438 \\
3.4109 \\
4.0948\end{array}$ & $\begin{array}{l}6.1706 \\
6.4947 \\
6.8784\end{array}$ & $\begin{array}{l}6.1862 \\
6.5096 \\
6.8925\end{array}$ & $\begin{array}{l}8.7973 \\
9.0275 \\
9.3074\end{array}$ & $\begin{array}{l}11.0065 \\
11.1914 \\
11.4183\end{array}$ & $\begin{array}{l}11.0719 \\
11.2557 \\
11.4813\end{array}$ \\
\hline FFCF & $\begin{array}{l}100 \\
500 \\
1000\end{array}$ & $\begin{array}{l}2.0523 \\
2.8841 \\
3.6676\end{array}$ & $\begin{array}{l}3.9254 \\
4.4176 \\
4.9646\end{array}$ & $\begin{array}{l}5.8134 \\
6.1564 \\
6.5600\end{array}$ & $\begin{array}{l}6.9810 \\
7.2691 \\
7.6139\end{array}$ & $\begin{array}{l}7.5194 \\
7.7876 \\
8.1103\end{array}$ & $\begin{array}{l}10.0809 \\
10.2825 \\
10.5290\end{array}$ \\
\hline CCSC & $\begin{array}{l}100 \\
500 \\
1000\end{array}$ & $\begin{array}{l}5.8824 \\
6.2216 \\
6.6212\end{array}$ & $\begin{array}{l}9.8819 \\
10.0874 \\
10.3386\end{array}$ & $\begin{array}{l}10.5168 \\
10.7102 \\
10.9471\end{array}$ & $\begin{array}{l}13.9569 \\
14.1031 \\
14.2838\end{array}$ & $\begin{array}{l}15.7839 \\
15.9133 \\
16.0736\end{array}$ & $\begin{array}{l}17.0247 \\
17.1448 \\
17.2937\end{array}$ \\
\hline
\end{tabular}


Table 8, continued

\begin{tabular}{lllllccc}
\hline B.C & $\mathrm{K}_{1}$ & \multicolumn{5}{c}{ Mode number } \\
\cline { 3 - 7 } & & 1st & 2nd & 3rd & 4th & 5th \\
\hline CSSS & 100 & 5.2997 & 8.9436 & 9.4994 & 12.6946 & 14.3813 \\
& 500 & 5.6739 & 9.1702 & 9.7131 & 12.8552 & 14.5233 \\
& 1000 & 6.1094 & 9.4458 & 9.9737 & 13.0532 & 14.6988 \\
FFFF & 100 & 1.0132 & 3.4292 & 3.4292 & 4.9146 & 7.1491 & 15.6382 \\
& 500 & 2.2656 & 3.9831 & 3.9831 & 5.3159 & 7.4307 & 7.1959 \\
& 1000 & 3.2041 & 4.5823 & 4.5823 & 5.7785 & 7.7683 & 7.8114 \\
\hline
\end{tabular}

$$
\begin{aligned}
& \mathbf{K}_{F}=\int_{S} \mathbf{H}_{I}^{\mathrm{T}} \mathbf{D}_{F} \mathbf{H}_{J} \mathrm{~d} S, \\
& \mathbf{M}=\int_{V} \rho \mathbf{N}_{I}^{\mathrm{T}} \mathbf{N}_{J} \mathrm{~d} V, \\
& \tilde{\mathbf{K}}=\int_{\Gamma_{u}} \boldsymbol{\Psi}_{I}^{\mathrm{T}} \boldsymbol{\alpha} \boldsymbol{\Psi}_{J} \mathrm{~d} \Gamma,
\end{aligned}
$$

where

$$
\begin{aligned}
& \mathbf{B}=\mathbf{L}_{p s} \phi_{I}=\left\{\begin{array}{c}
-\phi_{I, x x} \\
-\phi_{I, y y} \\
-2 \phi_{I, x y}
\end{array}\right\}, \\
& \mathbf{H}=\mathbf{L}_{f s} \phi_{I}=\left\{\begin{array}{c}
\phi_{I} \\
\phi_{I, x} \\
\phi_{I, y}
\end{array}\right\} \\
& \mathbf{N}=\mathbf{L}_{u} \phi_{I}=\left\{\begin{array}{c}
-z \phi_{I, x} \\
-z \phi_{I, y} \\
\phi_{I}
\end{array}\right\},
\end{aligned}
$$

$\Psi_{I}$ depends on boundary condition. For clamped boundary condition, $\Psi_{I}$ is defined as follows,

$$
\boldsymbol{\Psi}_{I}=\left\{\begin{array}{c}
\phi_{I} \\
\phi_{I, n}
\end{array}\right\},
$$

For simply support boundary condition it is defined as follows,

$$
\mathbf{\Psi}_{I}=\left\{\begin{array}{c}
\phi_{I} \\
0
\end{array}\right\},
$$

Assuming a harmonic vibration form for the plate, the deflection vector $\mathbf{U}$ can be expressed as:

$$
\mathbf{U}=\overline{\mathbf{U}} e^{i \omega \mathrm{t}},
$$

where $\overline{\mathbf{U}}$ is the amplitude of the vibration and $\omega$ is the circular frequency. Substituting Eq. (63) into Eq. (52), the following eigenvalue equation is obtained:

$$
\left(\mathbf{K}-\omega^{2} \mathbf{M}\right) \overline{\mathbf{U}}=0,
$$

where $\omega^{2}$ is the eigenvalue and represents the square of circular frequency of transverse vibration and $\overline{\mathbf{U}}$ is the eigenvector that represents the vector of amplitude of transverse vibration. 


\section{Numerical results and discussion}

In this paper, quadratic basis is adopted for basis function $\mathbf{P}(\mathbf{x})$, scaling parameter $d_{\max }$ is chosen as 3.9 [27] and a $4 \times 4$ gauss integration scheme is used to perform the integration in computing the system matrixes. Also the Poisson ratio $v$ is assumed 0.3 . To show accuracy and applicability of the present method, a computer code in MATLAB was developed and some examples of square plates were solved. Different boundary conditions, foundation parameters and number of nodes were considered.

To ease comparison of results, the following dimensionless parameters are defined:

1- Natural frequency of plate resting on elastic foundation,

$$
\bar{\omega}=\frac{\omega a^{2}}{\pi^{2}} \sqrt{\frac{\rho h}{D}},
$$

where $D$ is the bending rigidity of plate and defined as follows:

$$
D=\frac{\mathrm{Eh}^{3}}{12\left(1-v^{2}\right)},
$$

2- Foundation parameters,

$$
\begin{aligned}
& K_{1}=\frac{k_{f} a^{4}}{D}, \\
& K_{2}=\frac{G_{f} a^{2}}{D},
\end{aligned}
$$

\subsection{Free vibration of a square plate resting on Winkler's type elastic foundation}

The convergency and accuracy of the element free Galerkin method in vibration analysis of thin plates resting on Winkler elastic foundation is studied for different foundation parameters, various types of boundary conditions and different numbers of modes of vibration. In Table 1 the convergency and accuracy of the first and sixth natural frequencies are shown for different types of boundary conditions and two values of Winkler foundation stiffness. As it is seen, the results are convergent and are in very good agreement with the exact solutions of Ref [3]. Also, it is obvious from Table 1 that when the plate edge boundary condition is clamped or the vibrational results of the sixth mode of vibration would be obtained, more nodes should be used in order to achieve accurate results. In Table 2, the convergency of the first six modes of vibration is studied for a square SSSS plates resting on Winkler elastic foundation and as it is seen the convergency is monotonic. Also the results of the first four modes of the frequency are compared with the numerical solutions obtained by Ref [1,6,7] and good consistency is shown. It is seen that, using $5 \times 5$ number of nodes gives very accurate solution for the fundamental frequency and by increasing the number of mode of vibration, more nodes are needed in order to obtain precise results.

\subsection{Free vibration of a square plate resting on Pasternak's type elastic foundation}

In the end vibration of square thin plates resting on Pasternak's type elastic foundation is investigated. To show convergency and accuracy of the method for this type of foundation, in Table 3 the first and sixth natural frequencies of square plates with different foundation parameters and various types of boundary conditions are calculated and compared with exact solutions of Ref [3]. As it is shown, the results are convergent and a very good agreement with Ref [3] is seen. In Table 4, the first six natural frequencies for a SSSS plate with different elastic foundation parameters and thickness ratios are calculated and compared with available results. As can be seen very good agreements is achieved in all cases. In Table 5 the first six natural frequencies for a CCCC plate are calculated and compared with available literature and good agreements is obtained.

In order to compare the Winkler's type of elastic foundation with Pasternak's, in Figs 4-6 the first nine natural frequencies of plates with these two foundations are shown. As can be seen, by increasing the mode number and the shear stiffness parameter of elastic foundation $\mathrm{K}_{2}$, differences between these two foundations increase.

The first six natural frequencies of a square plate resting on Pasternak foundation are presented in Tables 6-8 for all possible types of classical boundary conditions and various values of foundation parameters. 


\section{Conclusions}

In this paper Element Free Galerkin method has been used for free vibration analysis of thin plates resting on two-parameter type elastic foundation with all possible types of boundary condition. Accuracy of the solution was examined for different foundation parameters, thickness to span ratio and boundary conditions. It was found that the method has very good agreement with available literature regardless of different parameters even with small number of nodes. Applicability of the method was demonstrated with solution of square plates with general boundary conditions, and foundation parameters. The numerical results provide valuable information for engineers in design applications and may also serve as benchmarks for further reference.

\section{References}

[1] Y. Xiang, C.M. Wang and S. Kitipornchai, Exact vibration solution for initially stressed Mindlin plates on Pasternak foundation, International Journal of Mechanical Sciences 36 (1994), 311-316.

[2] M.H. Omurtag, A. Ozutok and A.Y. Akoz, Free vibration analysis of Kirchhoff plates resting on elastic foundation by mixed finite element formulation based on gateaux differential, International Journal for Numerical Methods in Engineering 40 (1997), 295-317.

[3] K.Y. Lam, C.M. Wang and X.Q. He, Canonical exact solutions for levy-plates on two-parameter foundation using green's functions, Engineering Structures 22 (2000), 364-378.

[4] H. Matsunaga, Vibration and stability of thick plates on elastic foundations, Journal of Engineering Mechanics ASCE 126 (2000), 27-34.

[5] H.S. Shen, J. Yang and L. Zhang, Free and forced vibration of Reissner-Mindlin plates with free edges resting on elastic foundation, Journal of Sound and Vibration 244 (2001), 299-320.

[6] D. Zhou, Y.K. Cheung, S.H. Lo and F.T.K. Au, Three-dimensional vibration analysis of rectangular thick plates on Pasternak foundation, International Journal for Numerical Methods in Engineering 59 (2004), 1313-1334.

[7] A.J.M. Ferreira, C.M.C. Roque, A.M.A. Neves, R.M.N. Jorge and C.M.M. Soares, Analysis of plates on Pasternak foundations by radial basis, Computational Mechanics 46 (2010), 79-803.

[8] J.J. Monaghan, An introduction to SPH, Computer Physics Communications 48 (1988), 89-96.

[9] S.N. Atluri and T. Zhu, A new meshless local Petrov-Galerkin (MLPG) approach in computational mechanics, Computational Mechanics 22 (1998), 117-127.

[10] W.K. Liu, S. Jun and Y.F. Zhang, Reproducing kernel particle methods, International Journal for Numerical Methods in Engineering 20 (1995), 1081-1106.

[11] T. Belytschko, Y.Y. Lu and L. Gu, Element-free Galerkin methods, International Journal for Numerical Methods in Engineering 37 (1994), $229-256$.

[12] G.R. Liu and Y.T. Gu, A local radial point interpolation method (LRPIM) for free vibration analyses of 2-d solids, Journal of Sound and Vibration 246 (2001), 29-46.

[13] G.R. Liu and J.G. Wang, A point interpolation meshless method based on radial basis functions, International Journal for Numerical Methods in Engineering 54 (2002), 1623-1648.

[14] J.T. Chen, I.L. Chen, K.H. Chen, Y.T. Yeh and Y.T. Lee, A meshless method for free vibration of arbitrarily shaped plates with clamped boundaries using radial basis function, Engineering Analysis with Boundary Elements 28 (2004), 535-545.

[15] J.T. Chen, Y.T. Lee, I.L. Chen and K.H. Chen, Mathematical analysis and treatment for the true and spurious eigenequations of circular plates in the meshless method using radial basis function, J Chinese Institute of Engineers 27 (2004), 547-561.

[16] W. Yan, W. Zhong-min and R. Miao, Element-free Galerkin method for free vibration of rectangular plates with interior elastic point supports and elastically restrained edges, Journal of Shanghai University (English Edition) 14 (2010), 187-195.

[17] X.L. Chen, G.R. Liu and S.P. Lim, An element free Galerkin method for the free vibration analysis of composite laminates of complicated shape, Composite Structures 59 (2003), 279-289.

[18] K.Y. Dai, G.R. Liu, K.M. Lim and X.L. Chen, A mesh-free method for static and free vibration analysis of shear deformable laminated composite plates, Journal of Sound and Vibration 269 (2004), 633-652.

[19] P. Lancaster and K. Salkauskas, Surfaces generated by moving least squares, methods, Mathematics of Computation 37 (1981), $141-158$.

[20] T. Belytschko, Y. Krongauz, M. Fleming, D. Organ and W.K.S. Liu, Smoothing and accelerated computations in the element free Galerkin method, Journal of Computational and Applied Mathematics 74 (1996), 111-126.

[21] J. Dolbow and T. Belytschko, An introduction to programming the meshless element free galerkin method, Archives of Computational Methods in Engineering 5 (1998), 207-242.

[22] T. Zhu and S.N. Atluri, A modified collocation method and a penalty formulation for enforcing the essential boundary conditions in the element free galerkin method, Computational Mechanic 21 (1998), 211-222.

[23] G.R. Liu and K.Y. Yang, A penalty method for enforcing essential boundary conditions in element free galerkin method, The Proceeding of the 3rd HPC Asia'98 Singapore (1998), 71-721.

[24] D. Hegen, Element-free galerkin methods in combination with finite element approaches, Computer Methods in Applied Mechanics and Engineering 135 (1996), 143-166.

[25] G.R. Liu and Y.T. Gu, Coupling of element free galerkin and hybrid boundary element methods using modified variational formulation, Computational Mechanics 26 (2000), 16-173.

[26] G.R. Liu, Mesh Free Methods: Moving Beyond the Finite Element Method, New York: CRC Press, 2002.

[27] P. Krysl and T. Belytschko, Analysis of thin plates by the element-free Galerkin method, Computational Mechanic 17 (1995), 26-35. 

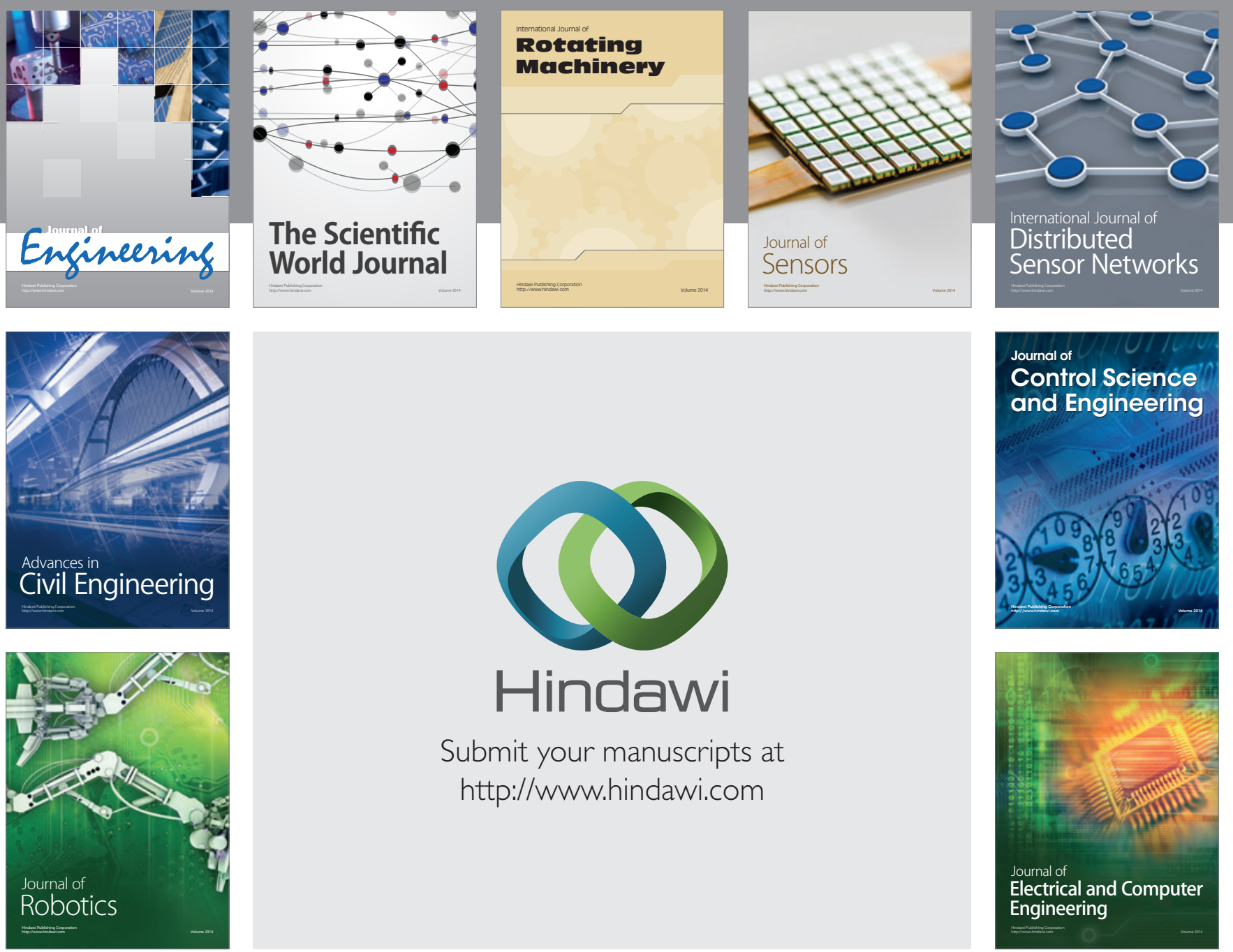

Submit your manuscripts at

http://www.hindawi.com
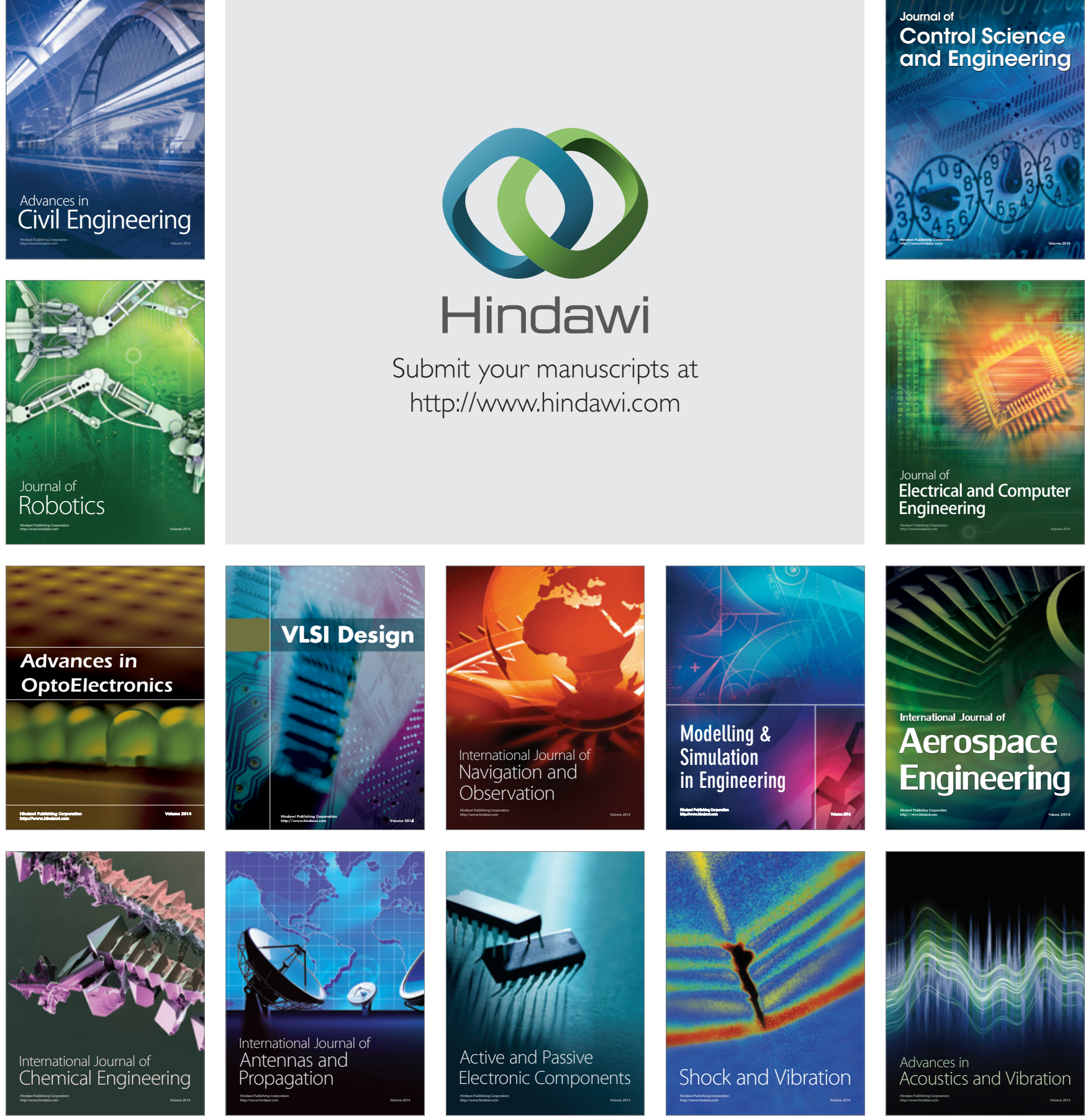